ESAIM: M2AN 47 (2013) 1037-1057

DOI: $10.1051 / \mathrm{m} 2 \mathrm{an} / 2012056$
ESAIM: Mathematical Modelling and Numerical Analysis

www.esaim-m2an.org

\title{
UNCERTAINTY QUANTIFICATION FOR DATA ASSIMILATION IN A STEADY INCOMPRESSIBLE NAVIER-STOKES PROBLEM
}

\author{
Marta D’Elia ${ }^{1}$ and Alessandro Veneziani ${ }^{1}$
}

\begin{abstract}
The reliable and effective assimilation of measurements and numerical simulations in engineering applications involving computational fluid dynamics is an emerging problem as soon as new devices provide more data. In this paper we are mainly driven by hemodynamics applications, a field where the progressive increment of measures and numerical tools makes this problem particularly upto-date. We adopt a Bayesian approach to the inclusion of noisy data in the incompressible steady Navier-Stokes equations (NSE). The purpose is the quantification of uncertainty affecting velocity and flow related variables of interest, all treated as random variables. The method consists in the solution of an optimization problem where the misfit between data and velocity - in a convenient norm - is minimized under the constraint of the NSE. We derive classical point estimators, namely the maximum a posteriori - MAP - and the maximum likelihood - ML - ones. In addition, we obtain confidence regions for velocity and wall shear stress, a flow related variable of medical relevance. Numerical simulations in 2-dimensional and axisymmetric 3-dimensional domains show the gain yielded by the introduction of a complete statistical knowledge in the assimilation process.
\end{abstract}

Mathematics Subject Classification. 76D06, 76M10, 62F15, 60H30.

Received December 24, 2011.

Published online June 13, 2013.

\section{INTRODUCTION}

Reliability of numerical methods is becoming a crucial issue as scientific computing has an increasing role in different fields of engineering and science. Many applied fields that resort to numerical simulations experience an increment of the amount of available data thanks to new measurement devices and efficient image processing techniques. This is the case of biomedicine and in particular hemodynamics (see e.g. [6]). As an example, Figure 1 [8] shows an MRI of the ascending aorta where blood velocity measurements have been collected. These data do not just represent a benchmark for validating numerical models, but can be profitably used for improving the quality of results. This motivates the introduction of data assimilation (DA) methods where the information provided by the observations is used to estimate variables and parameters of interest or for predicting statistical properties of computed variables $[1,22,25]$. In these terms, DA leads to the solution of an inverse problem whose input data is affected by uncertainty.

Keywords and phrases. Computational fluid dynamics, optimization, uncertainty quantification, statistical inverse problems, data assimilation, hemodynamics.

1 Dept. of Mathematics and Computer Science, Emory University, 400 Dowman Drive, Atlanta, GA 30322, USA.

mdelia2@mathcs.emory.edu; ale@mathcs.emory.edu 


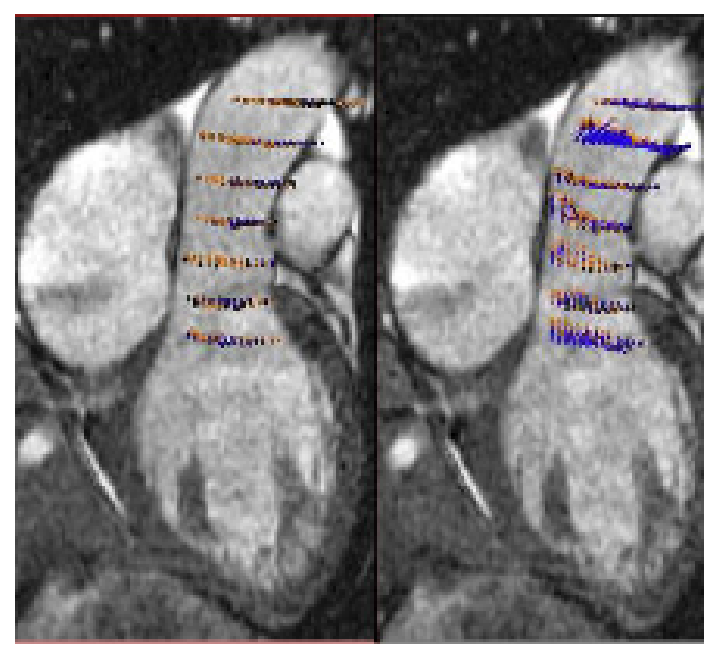

Figure 1. View of blood measured velocities in an MRI of the ascending aorta [8].

Problems related to DA have been addressed in several contexts (see e.g. [2]). Different approaches have been proposed in the literature, resorting to both deterministic and stochastic perspectives. We mention specifically variational methods (where DA is performed by minimizing a functional estimating the discrepancy between numerical results and measures, see e.g. $[2,14]$ ) and Kalman filter-based methods $[2,20]$.

DA for the Navier-Stokes equations in hemodynamics has been addressed by Hyes and collaborators [16] and Dwight [10] using a weighted least squares finite element method, by Bertoglio et al. [1] using an Unscented Kalman filter approach $[22,23]$ and by the authors in [7] using a variational approach. The term DA has often been used for time dependent phenomena. However, as done in [16], in this work we consider stationary phenomena and we refer to DA as a technique for the inclusion of data which are sparse in space and collected at the same time. We point out however that in $[5,7]$ this pseudo-steady approach has been used for tackling unsteady problems in a "discretize-in-time-then-optimize" setting.

In this paper we reconsider the procedure in the perspective of solving the associated statistical inverse problem. An extensive introduction to statistical inverse problems can be found in [19]. The purpose of statistical inversion is the prediction of stochastic features of the variables of interest in order to quantify the credibility of numerical methods and simulations. The prediction of the uncertainty affecting the variables is based on the knowledge of (i) the measurement process; (ii) deterministic models of the unknowns [19]. The main goal here is to obtain a quantification of the uncertainty induced by any probabilistic knowledge on the noise and on the variables we use as control function for the minimization of the misfit. The result is a reformulation of our procedure to the computation of estimators based on a Bayesian approach. As a matter of fact, even though we resort to an equivalent mathematical formulation of the deterministic case, the introduction of a priori knowledge improves the reliability of the assimilation.

In addition to the variational formulation, here we also also consider spread estimators in order to determine confidence regions for the velocity and the wall shear stress (WSS - i.e. the stress exerted by the fluid on the wall), which is a quantity of primary medical interest.

The computation of the estimator relies upon the assumption of linear problems, however it is worth noticing that we compute an error estimator for the nonlinear NSE, based on an iterative approach. A Newton-like linearization procedure, yet proposed for the completely deterministic assimilation, results to be numerically convergent for the estimation of the nonlinear case.

The outline of the paper is as follows. 
In Section 1 we summarize the deterministic formulation of the problem proposed in [7]. In Section 2 we introduce the mathematical formulation of the Bayesian method applied to the linearized NSE and we extend the formulation to the nonlinear case using the algorithm for the nonlinear equations introduced in Section 1. Then, we present point estimators, namely the maximum a posteriori (MAP) and the maximum likelihood (ML) estimators. Successively, we introduce spread estimators (confidence regions) based on the PDF of the normal stress on the inflow boundary. In Section 2.4 we re-consider a regularization technique based on interpolation introduced in [7] and show how it can be adjusted to the present framework for the ML estimator. In Section 3 we present numerical results in correspondence of 2-dimensional and axisymmetric 3-dimensional geometries with the purpose of comparing statistical estimators with those proposed in [7]. Also, we draw confidence regions for velocity and WSS. Concluding remarks and guidelines for future work can be found in Section 4 .

In Appendix we recall basic statistical notation and properties of normal random vectors which are useful for our application.

\section{Deterministic BACKGROUnd}

Let us denote by $\Omega$ a domain in $\mathbb{R}^{d}(d=2,3)$. We assume (see Figs. 1 and 2 ) that the domain of interest $\Omega$ features an inflow boundary $\Gamma_{\mathrm{in}}$, an outflow boundary $\Gamma_{\text {out }}$ and the physical wall of the vessel $\Gamma_{\text {wall }} . \Gamma_{\mathrm{in}}$ and $\Gamma_{\text {out }}$ can possibly consist of several sections, like in artery bifurcations. Variables of interest are velocity $\mathbf{u}(\mathbf{x}) \in$ $\mathbf{H}_{\Gamma_{\text {wall }}}^{1}(\Omega)=\left\{\mathbf{w} \in \mathbf{H}^{1}:\left.\mathbf{w}\right|_{\Gamma_{\text {wall }}}=\mathbf{0}\right\}$ and pressure $p(\mathbf{x}) \in L^{2}(\Omega)$ which are assumed to obey the incompressible NSE. Also, we assume to have $N_{s}$ velocity measures $\mathbf{d}$ available at some $\operatorname{sites}^{2} \mathbf{x}_{i}^{m} \in \Omega$, for $i=1, \ldots, N_{s}$. We use DA to estimate the velocity field combining these measures with the state equations. In [7] we solved this problem using a variational approach in a deterministic way. We choose the inflow normal stress, $\mathbf{h}(\mathbf{x}) \in \mathbf{H}^{-1 / 2}$, to be the control variable; this choice is arbitrary, however, we may notice that an accurate knowledge of inflowoutflow boundary conditions is quite problematic in real applications, since these are "artificial" boundaries (see $[17,29,30]$ ), so we cannot in general rely on their measures. For this reason, they have been used for the prescription of defective boundary conditions in $[11,12])$ in a variational setting.

The steady control problem reads: find $\mathbf{h} \in \mathbf{H}^{-1 / 2}\left(\Gamma_{\text {in }}\right)$ that minimizes

$$
\begin{aligned}
& \mathcal{J}(\mathbf{u}, \mathbf{h})=\operatorname{dist}(f(\mathbf{u}), \mathbf{d})+\mathcal{R}(\mathbf{h}) \\
& \text { s.t. }\left\{\begin{array}{lc}
-\nu \nabla \cdot\left(\nabla \mathbf{u}+\nabla \mathbf{u}^{\mathrm{T}}\right)+(\mathbf{u} \cdot \nabla) \mathbf{u}+\nabla p=\mathbf{s} \text { in } \Omega, \\
\nabla \cdot \mathbf{u}=0 & \text { in } \Omega, \\
\mathbf{u}=\mathbf{0} & \text { on } \Gamma_{\text {wall }}, \\
-\nu\left(\nabla \mathbf{u}+\nabla \mathbf{u}^{\mathrm{T}}\right) \mathbf{n}+p \mathbf{n}=\mathbf{h} & \text { on } \Gamma_{\mathrm{in}}, \\
-\nu\left(\nabla \mathbf{u}+\nabla \mathbf{u}^{\mathrm{T}}\right) \mathbf{n}+p \mathbf{n}=\mathbf{g} & \text { on } \Gamma_{\text {out }} .
\end{array}\right.
\end{aligned}
$$

Here, $\operatorname{dist}(\cdot, \cdot)$ is a distance measure, $f$ is an observation operator for the velocity field; $\mathbf{s} \in \mathbf{H}^{-1}(\Omega)$ is the source term; $\mathbf{g} \in \mathbf{H}^{-1 / 2}\left(\Gamma_{\text {out }}\right)$ is the Neumann data on the outflow section; $\mathcal{R}$ is a regularization term added to improve properties of the functional to be minimized, as we will specify later; $\nu$ is the kinematic viscosity. The choice of homogeneous Dirichlet boundary conditions on $\Gamma_{\text {wall }}$ is due to the fact that we consider fixed geometries. A (challenging) follow-up of the present work will be the extension of the assimilation procedure to fluid-structure interaction problems.

For the numerical solution of problem (1.1) we use a discretize then optimize technique relying on the finite element (FE) method. At first, we focus on the linearized state equations, replacing $(\mathbf{u} \cdot \nabla) \mathbf{u}$ with $\boldsymbol{\beta} \cdot \nabla \mathbf{u}$, being $\boldsymbol{\beta}$

\footnotetext{
${ }^{2}$ Notice that we use the word "sites" for the location of measurements, as opposed to the word "nodes" for points where velocities are computed. In general sites and nodes are different, but we do not exclude that they could partially coincide.
} 


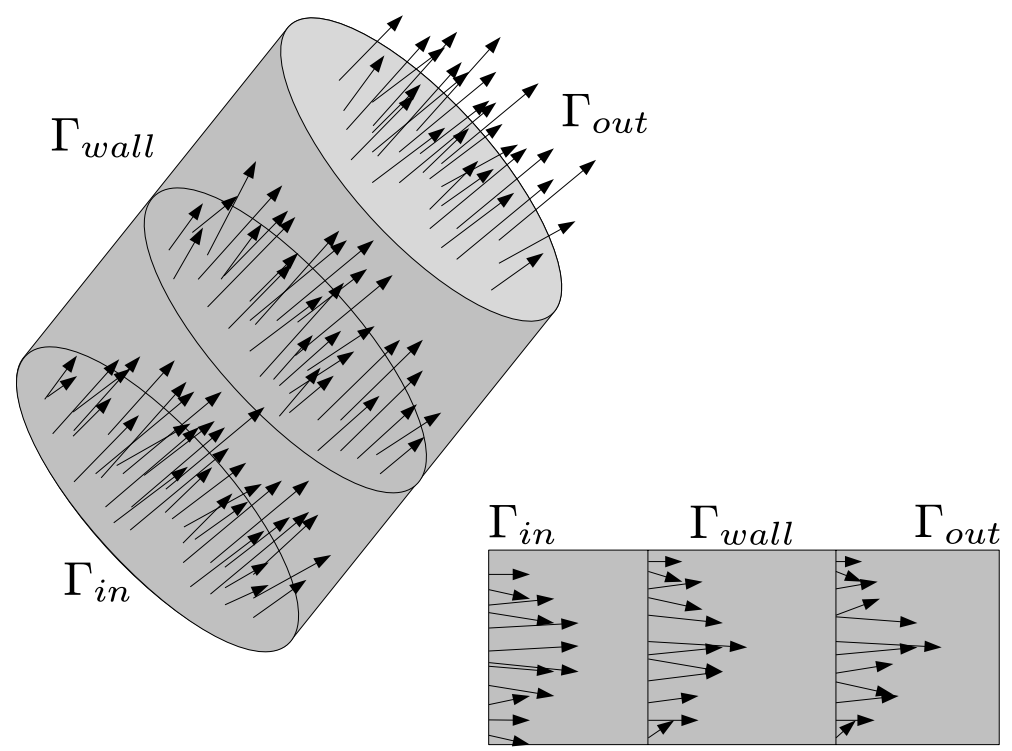

Figure 2. Possible 3-dimensional and 2-dimensional domains with associated measured sparse data.

a known advection field. We introduce the following notations,

$$
\mathbf{V}=\left[\begin{array}{l}
\mathbf{U} \\
\mathbf{P}
\end{array}\right], \quad \mathrm{S}=\left[\begin{array}{cc}
\mathrm{C}+\mathrm{A} & \mathrm{B}^{\mathrm{T}} \\
\mathrm{B} & \mathrm{O}
\end{array}\right]
$$

Here $\mathbf{U} \in \mathbb{R}^{N_{u}}$ and $\mathbf{P} \in \mathbb{R}^{N_{p}}$ are the discretization of velocity and pressure belonging to inf-sup compatible FE spaces; $N_{u}$ and $N_{p}$ are the number of degrees of freedom (DOFs) of velocity and pressure. $\mathbf{H} \in \mathbb{R}^{N_{\text {in }}}$ is the discretization of the control variable $\mathbf{h} ; N_{\text {in }}$ is the number of DOFs of the velocity on $\Gamma_{\text {in }}$.

We let C, A $\in \mathbb{R}^{N_{u}, N_{u}}$ and $\mathrm{B} \in \mathbb{R}^{N_{p}, N_{u}}$ be the discretization of the diffusion, advection and divergence operators. We let $\mathrm{Q} \in \mathbb{R}^{d N_{s}, N_{u}}$ be a matrix designed such that $[\mathrm{Q} \mathbf{U}]_{i}$ is the numerical solution evaluated at the data sites. We extend the selection matrix to pressure DOFs, introducing the matrix $\mathrm{D}=[\mathrm{Q} O]$. Should measurements on the pressure be available, the second block of $\mathrm{D}$ would be clearly non-zero. We let $\mathrm{R}_{i n, u} \in \mathbb{R}^{N_{\mathrm{in}}, N_{u}}$ be a restriction matrix which selects the DOFs of the velocity on $\Gamma_{\mathrm{in}}$. Again, the generalization of $\mathrm{R}_{i n, u}$ to velocity and pressure DOFs is $\mathrm{R}_{\text {in }}=\left[\mathrm{R}_{i n, u} \mathrm{O}\right]$, that belongs to $\mathbb{R}^{N_{\text {in }}, N_{u}+N_{p}}$. We let $\mathrm{M}_{\text {in }} \in \mathbb{R}^{N_{\text {in }}, N_{\text {in }}}$ be the discretization of the mass operator restricted to inlet boundary nodes.

The discretized problem reads: find $\mathbf{H}$ that minimizes

$$
\begin{aligned}
J(\mathbf{V}, \mathbf{H}) & =\frac{1}{2}\|\mathrm{D} \mathbf{V}-\mathbf{d}\|_{2}^{2}+\frac{\alpha}{2}\|\mathrm{~L} \mathbf{H}\|_{2}^{2} \\
\text { s.t. } \quad \mathrm{S} \mathbf{V} & =\mathrm{R}_{\mathrm{in}}^{\mathrm{T}} \mathrm{M}_{\mathrm{in}} \mathbf{H}+\mathbf{F} .
\end{aligned}
$$

Here, $\mathbf{F}$ denotes the discretization of the forcing term $\mathbf{s}$. For $\alpha>0$, the term $\frac{\alpha}{2}\|\mathrm{LH}\|_{2}^{2}$ has a regularization purpose (Tikhonov regularization, see [15]). $\mathrm{L}$ is a full rank matrix so that $\mathrm{L}^{\mathrm{T}} \mathrm{L}$ is positive definite. This optimization problem can be solved by the reduced Hessian method; the system of first order necessary conditions induced by the Lagrangian functional is in fact solved by block substitution yielding the following problem:

$$
\left(\mathrm{Z}^{\mathrm{T}} \mathrm{Z}+\alpha \mathrm{L}^{\mathrm{T}} \mathrm{L}\right) \mathbf{H}=\mathrm{Z}^{\mathrm{T}}\left(\mathbf{d}-\mathrm{DS}^{-1} \mathbf{F}\right),
$$

where $Z=D S^{-1} R_{\text {in }}^{T} M_{i n}$ [7]. This matrix, defined as $Z=\frac{d(D V)}{d \mathbf{H}}$, is the so-called sensitivity matrix [24]. Spectral properties of $Z$ determine the conditioning and well-posedness of the problem. In [7] we studied the 
non-singularity of system (1.4) and derived sufficient conditions for the existence of a unique minimizer. We summarize the main result in the following proposition.

Proposition 1.1. Sufficient conditions for the non-singularity of the reduced Hessian matrix are either:

1. $\alpha>0$, or

2. for $\alpha=0, \operatorname{Null}(\mathrm{D}) \cap \operatorname{Range}\left(\mathrm{S}^{-1} \mathrm{R}_{\mathrm{in}}^{\mathrm{T}} \mathrm{M}_{\mathrm{in}}\right)=\{\mathbf{0}\}$.

Condition 2. is satisfied when the selection matrix $\mathrm{Q}$ is designed such that its restriction $\mathrm{Q}_{r}$ to a subset of sites on $\Gamma_{\text {in }}$ fulfills $\mathrm{Q}_{r}\left(I-\mathrm{R}_{i n, u}^{\mathrm{T}} \mathrm{R}_{i n, u}\right)=\mathrm{O}$ and $\operatorname{rank}\left(\mathrm{Q}_{r}\right)=N_{\text {in }}$.

For treating the nonlinear problem we developed an iterative procedure. Let $\mathbf{U}_{k}$ be a given guess for the velocity field at iteration $k+1$ and $\mathbf{V}_{k}$ the corresponding vector of velocity and pressure. At each step we solve

$$
\begin{aligned}
& \min _{\mathbf{H}} \frac{1}{2}\left\|\mathrm{D} \mathbf{V}_{k+1}-\mathbf{d}\right\|_{2}^{2}+\frac{\alpha}{2}\left\|\mathbf{L H}_{k+1}\right\|_{2}^{2} \\
& \text { s.t. } \quad \mathrm{S}_{k} \mathbf{V}_{k+1}=\mathrm{R}_{\mathrm{in}}^{\mathrm{T}} \mathrm{M}_{\mathrm{in}} \mathbf{H}_{k+1}+\mathbf{F}_{k}
\end{aligned}
$$

up to fulfillment of a convergence criterion, typically $\left\|\mathbf{V}_{k}-\mathbf{V}_{k+1}\right\| \leq \delta$, being $\delta$ a user defined tolerance. In [7] we showed numerical evidence of the convergence of the algorithm, even though a rigorous proof of convergence is still missing. Here, we use the following notation

$$
\mathrm{S}_{k}=\left[\begin{array}{cc}
\mathrm{C}+\mathrm{A}_{k} & \mathrm{~B}^{\mathrm{T}} \\
\mathrm{B} & \mathrm{O}
\end{array}\right], \quad \text { and } \quad \mathbf{F}_{k}=\mathbf{F}+w \mathbf{Y}_{k}
$$

$\mathrm{A}_{k}$ comes from the discretization of $\left(\overline{\mathbf{u}}_{k} \cdot \nabla\right) \mathbf{u}_{k+1}+w\left(\mathbf{u}_{k+1} \cdot \nabla\right) \overline{\mathbf{u}}_{k}$, while $\mathbf{Y}_{k}$ is the discretization of $\left(\overline{\mathbf{u}}_{k} \cdot \nabla\right) \overline{\mathbf{u}}_{k}$; $\overline{\mathbf{u}}_{k}$ is defined as $\vartheta \mathbf{u}_{k-1}+(1-\vartheta) \mathbf{u}_{k}$. Here, $w \in[0,1]$, and $\vartheta \in[0,1]$ are relaxation parameters. Note that when $\vartheta=0$ and $w=0$ we have the Picard method while for $\vartheta=0$ and $w=1$ we have the Newton one.

Numerical results in [7] show that our procedure is an effective and robust noise filtering method. The discretization error with respect to the exact solution, decreases as more data are available: the convergence rate with respect to the number of sites, $N_{s}$, is of the order of $N_{s}^{-0.5}$. The sample mean of the computed velocity over $N_{r}$ noise realizations converges to the noise-free solution with rate $N_{r}^{-0.5}$, as expected by theory. Also, we observed a linear behavior of the error with respect to the amount of noise.

\section{Statistical Assimilation}

In formulating the statistical inverse problem (see e.g. $[4,19]$ ) we assume to deal with discrete variables, all treated as random. This means that the entities involved are PDFs as well as the result of the assimilation.

In the following sections the bold variables denote random vectors while with capital plain variables we indicate a corresponding specific realization. We introduce the random variable $\mathbf{H}$ which describes the normal stress of the fluid at the inflow section; $\mathbf{M}$ is the random variable that describes the measures and $\varepsilon$ is the noise perturbing the measurements. We let $\pi_{\mathrm{pr}}(H)$ be the prior $\mathrm{PDF}$ of $\mathbf{H}$, and $\pi_{\text {noise }}(\varepsilon)$ the one of $\varepsilon$; these distributions are assumed to be known. The likelihood function $\pi(M \mid H)$ denotes the PDF of the measurement conditioned on a realization of $\mathbf{H}$. The posterior distribution $\pi(H \mid M)$, i.e. the distribution of $\mathbf{H}$ conditioned on a realization of $\mathbf{M}$, is denoted by $\pi_{\text {post }}(H)$. The purpose of the Bayesian procedure is to estimate the posterior exploiting the Bayes formula

$$
\pi(H \mid M)=\pi_{\text {post }}(H)=\frac{\pi(M \mid H) \pi_{\mathrm{pr}}(H)}{\pi(M)}
$$

where $\pi(M)$ is the PDF of the measures. Since we are interested in finding $\mathbf{H}$, that maximizes the posterior distribution, the denominator does not affect the optimization process and we can simply consider the relation

$$
\pi(H \mid M)=\pi_{\text {post }}(H) \propto \pi(M \mid H) \pi_{\text {pr }}(H) .
$$


Let us consider the linear model that relates $\mathbf{H}$ and $\mathbf{M}$,

$$
\mathrm{ZH}+\varepsilon=\mathbf{M} .
$$

Here, $\mathrm{Z}=\mathrm{DS}^{-1} \mathrm{R}_{\mathrm{in}} \mathrm{M}_{\mathrm{in}}$ is the matrix introduced in Section 1, which relates the random variable representing the velocity measures to the variable $\mathbf{H}$ in the case of the linearized NSE. The random variable $\varepsilon$ accounts for the discrepancy between the noise-free observation ZH and the actual measure M. Models in the form (2.3) are referred to as additive noise and they are used with the assumption of mutual independence of $\mathbf{H}$ and $\varepsilon$. This assumption implies that the PDF of $\varepsilon$ is independent of any realization of $\mathbf{H}$, say $\bar{H}$. Hence, the likelihood function can be expressed as

$$
\pi(M \mid \bar{H})=\pi_{\text {noise }}(M-\mathrm{Z} \bar{H}) .
$$

If we consider $M=\mathbf{d}$, a specific realization of $\mathbf{M}$, we rewrite (2.2) as

$$
\pi_{\text {post }}(H) \propto \pi_{\text {noise }}(\mathbf{d}-\mathrm{Z} H) \pi_{\mathrm{pr}}(H) .
$$

Notice that the choice of model (2.3) makes our method substantially different from the one proposed by Heys in [16]. In fact, in our case (2.3) is a constraint and the numerical solution satisfies the state equations. In [16] the assimilation is formulated as an unconstrained optimization problem where the state equations, together with a weighted misfit between velocity and data, are part of the functional to be minimized; in [10] this approach is reinterpreted in a Bayesian perspective, where the fulfillment of the Navier-Stokes problem is associated with the prior probability density function. In this way, the physical constraints are forced in a "weak" sense.

When we consider in particular Gaussian random variables we can derive an explicit form for $\pi_{\text {post }}(H)$. We introduce the PDFs of $\mathbf{H}$ and $\varepsilon$, according to the notation reported in the appendix:

$$
\begin{aligned}
& \pi_{\mathrm{pr}}(H) \propto \exp \left\{-\frac{1}{2}\left(H-H_{0}\right)^{\mathrm{T}} \Sigma_{\mathrm{pr}}^{-1}\left(H-H_{0}\right)\right\}, \\
& \pi_{\text {noise }}(\varepsilon) \propto \exp \left\{-\frac{1}{2}\left(\varepsilon-\varepsilon_{0}\right)^{\mathrm{T}} \Sigma_{\text {noise }}^{-1}\left(\varepsilon-\varepsilon_{0}\right)\right\},
\end{aligned}
$$

where $H_{0}$ and $\varepsilon_{0}$ are the expectation values and $\Sigma_{\mathrm{pr}}$ and $\Sigma_{\text {noise }}$ are the correlation matrices for $\mathbf{H}$ and $\varepsilon$ respectively. In the Gaussian assumption, using Theorem 3.7, Chapter 3 of [19], we can write (2.5) as

$$
\pi_{\text {post }}(H) \propto \exp \left\{-\frac{1}{2}\left(H-H_{\text {post }}\right)^{\mathrm{T}} \Sigma_{\text {post }}^{-1}\left(H-H_{\text {post }}\right)\right\} ;
$$

where the correlation matrix and the expectation value read

$$
\begin{aligned}
\Sigma_{\text {post }} & =\left(\Sigma_{\text {pr }}^{-1}+\mathrm{Z}^{\mathrm{T}} \Sigma_{\text {noise }}^{-1} \mathrm{Z}\right)^{-1}, \\
H_{\text {post }} & =\Sigma_{\text {post }}\left(\mathrm{Z}^{\mathrm{T}} \Sigma_{\text {noise }}^{-1}\left(\mathbf{d}-\varepsilon_{0}\right)+\Sigma_{\text {pr }}^{-1} H_{0}\right) \Rightarrow \\
\left(\Sigma_{\text {pr }}^{-1}+\mathrm{Z}^{\mathrm{T}} \Sigma_{\text {noise }}^{-1} \mathrm{Z}\right) H_{\text {post }} & =\mathrm{Z}^{\mathrm{T}} \Sigma_{\text {noise }}^{-1}\left(\mathbf{d}-\varepsilon_{0}\right)+\Sigma_{\text {pr }}^{-1} H_{0} .
\end{aligned}
$$

Using this result one can calculate point and spread estimates.

\subsection{Iterative estimators for the nonlinear NSE}

A possible extension to the nonlinear case can be obtained by an iterative fixed-point argument, according to a linearize then optimize paradigm. The algorithm reads as follows. Given a guess for the velocity at iteration $k+1$, we find $H_{\text {post }, k+1}$ by solving

$$
H_{\text {post }, k+1}=\left(\Sigma_{\text {pr }}^{-1}+\mathrm{Z}_{k}^{\mathrm{T}} \Sigma_{\text {noise }}^{-1} \mathrm{Z}_{k}\right)^{-1}\left(\mathrm{Z}_{k}^{\mathrm{T}} \Sigma_{\text {noise }}^{-1}\left(\mathbf{d}-\varepsilon_{0}\right)+\Sigma_{\mathrm{pr}}^{-1} H_{0}\right)
$$


until a convergence criterion is satisfied, typically $\left\|U_{k}-U_{k+1}\right\| \leq \delta, \delta$ being a user defined tolerance and $U_{k}$ being the velocity vector associated with the normal stress $H_{\text {post }, k}$. Here, $\mathrm{Z}_{k}$ is defined as $\mathrm{Z}_{k}=\mathrm{DS}_{k}^{-1} \mathrm{R}_{\text {in }}^{\mathrm{T}} \mathrm{M}_{\mathrm{in}}$ where $\mathrm{S}_{k}$ si defined as in (1.6). Note that with this formulation $\mathbf{H}$ and $\mathbf{U}$, at each iteration, are related by a linear model and, for this reason, $\mathbf{U}$ can still be considered normally distributed. Also in this case, a rigorous proof of convergence of the iterative method is missing. Numerical experiments illustrated below, conducted with several geometries and different model parameters, show however that convergence is guaranteed tuning the regularization parameter; in our case this procedure is performed empirically.

\subsection{Statistical point estimators}

One of the most common point estimators is the maximum a posteriori (MAP) one. We let $H_{\mathrm{MAP}}$ be the MAP estimator for $\mathbf{H}$. It is defined as follows

$$
H_{\mathrm{MAP}}=\arg \max _{H} \pi_{\mathrm{post}}(H) .
$$

In the Gaussian assumption $H_{\mathrm{MAP}}$ corresponds to the expected value of the posterior distribution, $H_{\text {post }}$, as specified in (2.8).

We stress that the problem of finding $H_{\text {post }}$, solving system (2.8), is mathematically equivalent to problem (1.4), for the computation of the deterministic estimation of $\mathbf{H}$, with a proper choice of operators and norms in equation (1.3). Nevertheless, the estimate obtained in this way relies on a different level of information. This improves the reliability of the entire data assimilation procedure. In using the MAP estimator some knowledge on the distribution of $\mathbf{H}$ must be assumed. For this reason, we introduce "Gaussian smoothness" priors [19]; they correspond to prior models with structural information on $\mathbf{H}$. For instance, when (2.3) comes form the discretization of PDEs, it is a common assumption to consider $\mathbf{H}$ having smoothness properties (e.g. second order differentiability). In this work we choose $\Sigma_{\mathrm{pr}}^{-1}=\frac{1}{\sigma^{2}} \mathrm{~L}^{\mathrm{T}} \mathrm{L}$, where $\mathrm{L}$ corresponds to the discretization of the gradient. This highlights the role of the prior in (2.8), similar to the one of the Tikhonov regularization in the deterministic case.

Another common estimator is the maximum likelihood (ML) estimator; this is the value of $\mathbf{H}$ which is most likely to produce the data $\mathbf{d}$. It is defined as

$$
H_{\mathrm{ML}}=\arg \max _{H} \pi(M \mid H) .
$$

ML estimator can be in general not well defined. We introduce here his formal representation and discuss then its well-posedness.

For the Gaussian distribution, combining (2.4) and (2.6), this corresponds to solving

$$
\arg \max _{H} \exp \left\{-\frac{1}{2}\left(\mathbf{d}-\mathrm{Z} H-\varepsilon_{0}\right)^{\mathrm{T}} \Sigma_{\text {noise }}^{-1}\left(\mathbf{d}-\mathrm{Z} H-\varepsilon_{0}\right)\right\}
$$

or, equivalently

$$
\arg \min _{H}-\frac{1}{2}\left(\mathbf{d}-\mathrm{Z} H-\varepsilon_{0}\right)^{\mathrm{T}} \Sigma_{\text {noise }}^{-1}\left(\mathbf{d}-\mathrm{Z} H-\varepsilon_{0}\right),
$$

which leads to the solution of the following linear system

$$
\left(\mathrm{Z}^{\mathrm{T}} \Sigma_{\text {noise }}^{-1} \mathrm{Z}\right) H_{\mathrm{ML}}=\mathrm{Z}^{\mathrm{T}} \Sigma_{\text {noise }}^{-1}\left(\mathbf{d}-\varepsilon_{0}\right) .
$$

This is not a Bayesian estimator since it does not rely on the Bayes formula; it only requires the knowledge of stochastic features of the measurement process, i.e. the likelihood function. In addition, when the matrix of system (2.14) is singular the estimator is not well-defined. This happens when either $\Sigma_{\text {noise }}$ is singular or $\mathrm{Z}$ is rank-deficient. In the latter case, as proved in Proposition 1.1, singularity is due to data location. Under the assumptions of a non-singular noise correlation matrix and the conditions stated in Proposition 1.1, the 
ML estimator can be computed. Under these conditions, MAP and ML estimators are strictly related and the choice between the two basically depends on the level of prior knowledge on the variable of interest. By direct comparison of $H_{\mathrm{ML}}(2.14)$ and $H_{\text {post }}(2.8)$, the former actually corresponds to the case $\Sigma_{\mathrm{pr}}^{-1}=0$, i.e. when we reject our prior belief on $\mathbf{H}$. On the other hand the MAP estimation pulls the estimate towards the prior.

\subsection{Statistical spread estimators}

Beyond point estimates, much broader information is carried by the posterior distribution. In this section we define credibility regions for the velocity vector. Our goal is to have a measure of how likely the velocity, or one of its components, falls within a set of values, or, conversely, how large is the set of values corresponding to probability $\xi$ for the velocity to belong to such set.

Using properties of affine transformations of random variables and the theory of confidence regions for the multivariate normal distribution (see Appendix A) we introduce the PDF of $\mathbf{U}$ (the random variable associated with the discretized velocity) and credibility regions for components of the velocity vector in each DOF.

In both the linear and linearized cases, we have (see Eq. (1.3))

$$
\mathbf{U}=\mathrm{R}_{u}\left(\mathrm{~S}^{-1} \mathrm{R}_{\mathrm{in}}^{\mathrm{T}} \mathrm{M}_{\mathrm{in}} \mathbf{H}+\mathrm{S}^{-1} \mathbf{F}\right) ;
$$

where $R_{u}$ is the matrix that extracts the velocity, i.e. such that $R_{u} \mathbf{V}=\mathbf{U}$. From now on we set $\mathrm{T}=$ $\mathrm{R}_{u} \mathrm{~S}^{-1} \mathrm{R}_{\text {in }}^{\mathrm{T}} \mathrm{M}_{\mathrm{in}}$. This affine transformation maps the Gaussian variable $\mathbf{H} \sim \mathcal{N}\left(H_{\text {post }}, \Sigma_{\text {post }}\right)$ into the Gaussian variable $\mathbf{U} \sim \mathcal{N}\left(U, \Sigma_{U}\right)$ where $U=\mathrm{T} H_{\text {post }}+\mathrm{R}_{u} \mathrm{~S}^{-1} \mathbf{F}$ and $\Sigma_{U}=\mathrm{T} \Sigma_{\text {post }} \mathrm{T}^{\mathrm{T}}$. Here we assume for simplicity that $\mathbf{F}$ is not affected by uncertainties. We focus on credibility regions for the horizontal and vertical velocity in each DOF of the computational grid. If horizontal velocities are stored in the first half of the vector, discrete velocity in the generic DOF with index $i$ are located in $\left[\begin{array}{ll}\mathbf{U}_{i} & \mathbf{U}_{i+N_{u} / 2}\end{array}\right]^{\mathrm{T}} \in \mathbb{R}^{2}$, for $i=1, \ldots, N_{u} / 2$. Given the posterior density we can define the marginal densities of such 2 -dimensional vectors $\forall i$, which are still Gaussian (see property $\mathbf{P} 2$ in Appendix A) and distributed as $\mathcal{N}\left(\boldsymbol{\mu}_{i}, \Sigma_{i}\right)$, where

$$
\boldsymbol{\mu}_{i}=\left[\begin{array}{l}
U_{i} \\
U_{i+N_{u} / 2}
\end{array}\right] \text {, and } \Sigma_{i}=\left[\begin{array}{ll}
\Sigma_{U ; i, i} & \Sigma_{U ; i, i+N_{u} / 2} \\
\Sigma_{U ; i+N_{u} / 2, i} & \Sigma_{U ; i+N_{u} / 2, i+N_{u} / 2}
\end{array}\right] .
$$

Confidence intervals for $\mathbf{U}_{i}$ can be found using property P3 (see the Appendix A) for the 2-dimensional case. Since $\left(\mathbf{U}_{i}-\boldsymbol{\mu}_{i}\right)^{\mathrm{T}} \Sigma_{i}^{-1}\left(\mathbf{U}_{i}-\boldsymbol{\mu}_{i}\right)$ has a chi-squared distribution with 2 degrees of freedom, probability of $1-\xi$ can be assigned to the ellipsis $\left\{\mathbf{u}:\left(\mathbf{u}-\boldsymbol{\mu}_{i}\right)^{\mathrm{T}} \Sigma_{i}^{-1}\left(\mathbf{u}-\boldsymbol{\mu}_{\boldsymbol{i}}\right) \leq \chi_{2}^{2}(\xi)\right\}$ in the $(x, y)$ plane, where $\chi_{2}^{2}(\xi)$ denotes the upper $(100 \xi)$-th percentile of the distribution. Using this result, for a fixed value of $\xi$, we can draw confidence regions in the 2-dimensional plane as shown in Figure 3.

In this respect, spectral properties of the correlation matrix $\Sigma_{i}$ play a fundamental role; the eigenvectors of $\Sigma_{i}$ are in fact the directions of the main axes and the eigenvalues are a relative measure of the variability of the velocity components. In our investigation we consider a map over the computational grid of the max eigenvalue of the correlation matrix for the bivariate distribution; this corresponds to the maximum deviation form the mean in a confidence region of the $60 \%$ (corresponding to $\chi_{2}^{2}(1-0.6)$ ). Also, for selected DOFs, we draw $80 \%$ confidence regions (corresponding to $\chi_{2}^{2}(1-0.2)$ ).

Similar arguments can be used (see Sect. 3) in order to define statistical properties of the wall shear stress (WSS). The latter is the tangential component of the normal stress induced on the wall by the fluid,

$$
W S S=\mu\left(\nabla \mathbf{u}+\nabla^{T} \mathbf{u}\right) \cdot \mathbf{n}-\mathbf{n}^{T}\left(\mu\left(\nabla \mathbf{u}+\nabla^{T} \mathbf{u}\right) \cdot \mathbf{n}\right) \mathbf{n}
$$

where $\mathbf{n}$ is the outward unit vector. This quantity has a significant importance for monitoring the risk of atherosclerosis plaque development and his computation is used for the identification of regiones exposed to disease development. 


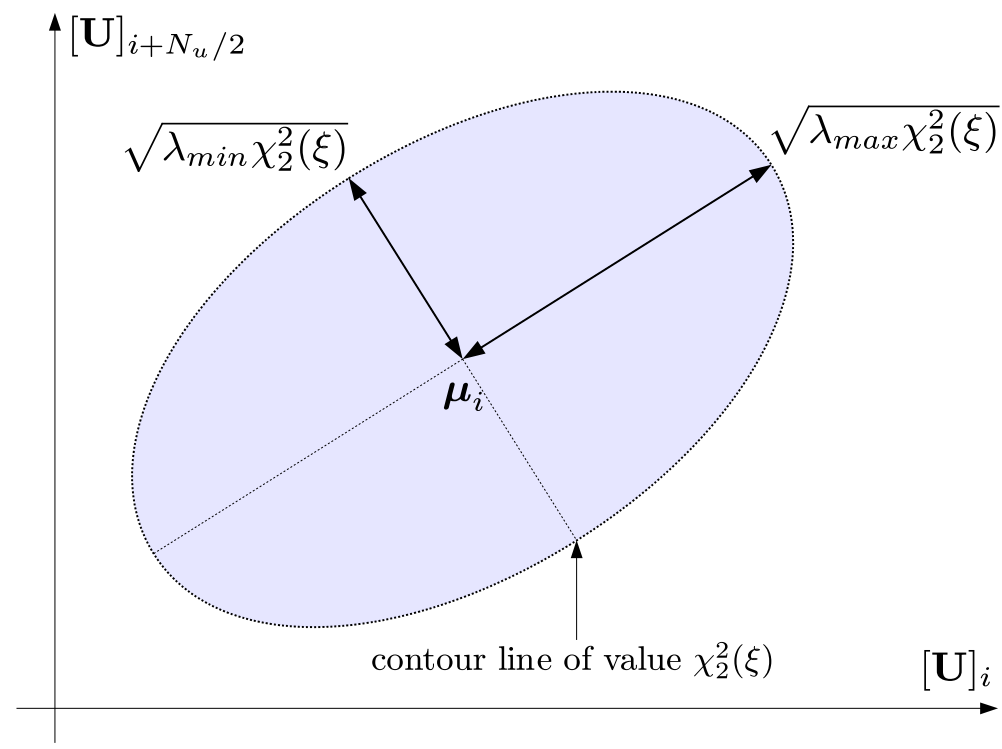

Figure 3. Confidence region associated with $\chi_{2}^{2}(\xi)$. The eigenvalues of $\Sigma_{i}$ are denoted by $\lambda_{1} \geq \lambda_{2}$.

\subsection{Regularization by interpolation}

In [7] on the basis of Proposition 1.1, we introduced a form of regularization alternative to Tikhonov techniques. As we have pointed out, a sufficient condition for the non-singularity of the problem without regularization is that the selection matrix, restricted to inflow boundary sites, should have rank $N_{\mathrm{in}}$. When this condition is not satisfied, we can build an interpolating function to be evaluated on the entire set of nodes on $\Gamma_{\mathrm{in}}$. If we assume data $\widetilde{\mathbf{d}}$ to be given, then, after interpolation, we can write $\mathbf{d}=\Pi \widetilde{\mathbf{d}}$, where $\Pi \in \mathbb{R}^{N_{s}+N_{\mathrm{in}}, N_{s}}$ is the interpolation matrix associated with piece-wise linear Lagrangian polynomials. In this way, using original and interpolated data the sufficient condition is fulfilled.

Results presented in [7] show that the interpolation is an effective parameter free form of regularization. However, it cannot be immediately extended to the present case. As a matter of fact, the interpolation process alters the noise distribution (specifically $\Sigma_{\text {noise }}$ ) introducing additional correlation among the data.

Let us assume to know the correlation matrix of the noise $\boldsymbol{\varepsilon}$. After interpolation we write $(2.3)$ as $\mathbf{Z H}+\boldsymbol{\eta}=\mathbf{M}$. Here, the random vector $\boldsymbol{\eta}=\Pi \varepsilon$ is associated with a new set of sites: the given ones and the DOFs on $\Gamma_{\mathrm{in}}$. In the Gaussian assumption, $\boldsymbol{\eta}$ is still normally distributed with mean $\Pi \varepsilon_{0}$ and correlation matrix $\Sigma_{\eta}=\Pi \Sigma_{\text {noise }} \Pi^{\mathrm{T}}$, see Appendix A. Note that $\Sigma_{\eta}$ is singular by construction, since the measures are now a linear dependent set. This circumstance makes $H_{\mathrm{ML}}$ in equation (2.14) not well-defined. A possible approach to recover well posedness in this case is to consider Gaussian smoothness priors. Here and in Section 3, we consider two other options.

(i) We filter out the null eigenvalues in $\Sigma_{\eta}$ by resorting to the pseudo-inverse $\Sigma_{\eta}^{+}$when solving the generic system $\Sigma_{\eta} \mathbf{x}=\mathbf{y}$.

(ii) We modify the correlation matrix, by pretending that interpolated data are independently and identically distributed. This is equivalent to setting the diagonal block of $\Sigma_{\eta}$ corresponding to DOFs on $\Gamma_{\text {in }}$ equal to $\sigma^{2}$ I, where I is the identity matrix of dimension $N_{\text {in }}$.

This clearly prevents singularity in the computation of the estimator, that in this case we will call "modified" ML. 
As we will see in the next section, the modification of the correlation matrix does not significantly compromise the accuracy of the statistical estimator, since the statistical information associated with interpolated data is redundant.

\section{NumericAl RESUlts}

We present numerical results obtained testing the Bayesian formulation on 2-dimensional and axisymmetric 3-dimensional geometries for both the linearized and nonlinear NSE. In order to test the effectiveness of this approach we compare the MAP and ML estimators with the estimates obtained using the formulation of Section 1. The estimator obtained by solving (1.4), i.e. without using any additional statistical information will be referred to as "deterministic" for brevity. Even though deterministic, MAP and ML estimators stem from an equivalent abstract formulation with different choices of the norms used for the constrained minimization, they obtain different results in terms of accuracy and number of iterations in the solution of linear systems. To this differences we are mainly concerned in this Section. We introduce an index of accuracy related to the velocity fields retrieved from $H_{\mathrm{MAP}}, H_{\mathrm{ML}}$ and $H_{\mathrm{det}}$; it is defined as $E_{U}=\frac{\left\|\mathbf{U}-\mathbf{U}_{e x}\right\|_{2}}{\left\|\mathbf{U}_{e x}\right\|_{2}}$, where $\mathbf{U}_{e x}$ is a discretized analytic solution, see equation (3.2), assumed known. We also define an average error over a set of noise realizations, $\bar{E}_{U}=\frac{1}{n} \sum_{i=1}^{n} \bar{E}_{U, i}$ where $E_{U, i}$ is associated with the $i$ th realization. In addition, we consider a measure of the gain, $\gamma$, in using statistical estimators as opposed to deterministic ones: $\gamma=1-\frac{\bar{E}_{U, \text { stat }}}{\bar{E}_{U, \text { det }}}$ where stat stands for either MAP or ML.

Statistical spread estimators for the velocity and for the WSS are derived and discussed too.

We test two possible sites configurations on $\Gamma_{\text {in }}$ and we discuss numerical results in terms of accuracy and statistical interpretation. We consider the solution of the linearized problem first; then, we treat the nonlinear case.

\subsection{Test cases setting}

\subsubsection{Implementation details}

We implement the FE method with the choice of compatible FE spaces P1bubble-P1 for velocity and pressure respectively. Numerical results are obtained with the $\mathrm{C}++\mathrm{FE}$ library $\mathrm{life \textrm {V } ^ { 3 }}$ and post-processed with ParaView ${ }^{4}$. For the solution of the linear system (2.8) we refer to the GMRESR method [9,31]; for the matrix S we consider the pressure-convection-diffusion preconditioner proposed by Kay in [21] and by Silvester et al. in $[27]$.

As smoothness prior we use $\Sigma_{\mathrm{pr}}^{-1}=\alpha \mathrm{L}^{\mathrm{T}} \mathrm{L}, \alpha \geq 0$, where $\mathrm{L}$ is the discrete gradient. This is a common choice for PDE constrained problems and it is not based on specific physical considerations. Nevertheless, we note that in hemodynamics applications some prior knowledge of the velocity and pressure distribution can be derived from the anatomy of the human body; this might be used to infer statistical properties of the control variable $\mathbf{H}$ using the relation between flow and boundary stress.

\subsubsection{Data generation}

We assume to have sites on $\Gamma_{\text {in }}$ and in $\Omega$ either on selected layers (featuring the configuration of Fig. 2) or uniformly distributed in the domain. At this preliminary stage data are synthetic, i.e. generated adding random noise to a known analytic solution. Moreover, we set the available data (the specific realization of $\mathbf{M}$ ) $\mathbf{d}=\mathrm{QU}_{e x}+\varepsilon, \mathrm{Q}$ being the selection matrix introduced in Section 1. Here, the Gaussian random vector $\varepsilon$ is generated coloring white noise: $\varepsilon=\mathrm{Gw}$, where $\mathbf{w} \sim \mathcal{N}(0, \mathrm{I})$ and $\mathrm{G}$ is such that $\mathrm{GG}^{\mathrm{T}}=\Sigma_{\varepsilon}$, the correlation matrix for the noise on sites, assumed known. We compute $\mathrm{G}$ as the Cholesky factor of $\Sigma_{\varepsilon}$.

\footnotetext{
3 http://www.lifev.org

${ }^{4}$ http://www. paraview.org
} 
TABLE 1. Accuracy results for statistical and deterministic solutions with data satisfying sufficient conditions for optimality.

\begin{tabular}{|c|cc|cc|ccc|}
\cline { 2 - 8 } \multicolumn{1}{c|}{} & \multicolumn{2}{|c|}{$H_{\text {det }}$} & \multicolumn{2}{c|}{$H_{\mathrm{ML}}$} & \multicolumn{3}{c|}{$H_{\mathrm{MAP}}$} \\
\hline IS & $\bar{E}_{U}$ & $\alpha$ & $\bar{E}_{U}$ & $\gamma$ & $\bar{E}_{U}$ & $\alpha$ & $\gamma$ \\
\hline 0 & 0.08077 & 0.1 & 0.06290 & $22 \%$ & 0.06296 & 0.001 & $22 \%$ \\
\hline 100 & 0.02956 & 0 & 0.02701 & $9 \%$ & 0.02882 & 0.10 & $3 \%$ \\
\hline 200 & 0.06868 & 0.15 & 0.03751 & $41 \%$ & 0.04853 & 0.15 & $29 \%$ \\
\hline 300 & 0.08837 & 0.15 & 0.02049 & $77 \%$ & 0.02662 & 0.2 & $70 \%$ \\
\hline
\end{tabular}

\subsubsection{The noise distribution}

In our test cases we consider $\varepsilon_{0}=\mathbf{0}$, which is a reasonable assumption in case of velocity data retrieved from MRIs (personal communication of Dr. Brummer, Emory CHOA). We assume that the noise correlation depends on the mutual distance between sites. In particular, we assume that close data highly affect each other, and that data significantly far from each other are almost independent. For these reasons, we consider an exponential decay with respect to the mutual distance. Formally,

$$
\left[\Sigma_{\text {noise }}\right]_{i j}=\left[\Sigma_{\varepsilon}\right]_{i j}=\sigma^{2} \exp \left\{-\frac{1}{l^{2}}\left\|\mathbf{x}_{i}^{m}-\mathbf{x}_{j}^{m}\right\|_{2}^{2}\right\} .
$$

Here, $l$ is a reference distance for the domain of interest and $\sigma$ is defined as $\sigma=\frac{U_{\max }}{\mathrm{SNR}}$, where $U_{\max }$ is the maximum magnitude of the velocity and SNR is the signal-to-noise ratio. Note that matrix $\Sigma_{\text {noise }}$ is symmetric positive definite.

\subsection{Point estimators}

Simulations presented in Test case $I, I I$ and $I I I$ are based on the 2-dimensional Navier-Stokes flow in the domain $\Omega=[-0.5,1.5] \times[0,2]$ whose exact solution, $\mathbf{u}_{e x}$, reads

$$
\left\{\begin{array}{l}
{[\mathbf{u}]_{1}(x, y)=1-\mathrm{e}^{\kappa x} \cos (2 \pi y), \quad[\mathbf{u}]_{2}(x, y)=\frac{\kappa}{2 \pi} \mathrm{e}^{\kappa x} \sin (2 \pi y),} \\
p(x, y)=\frac{1}{2} \mathrm{e}^{2 \kappa x}-\frac{1}{4 \kappa} \mathrm{e}^{2 \kappa} .
\end{array}\right.
$$

Here, the kinematic viscosity of the fluid is $\nu=0.035$. The adimensional parameter $\kappa$ is defined as $\kappa=$ $\frac{1}{2}\left(\nu^{-1}-\sqrt{\nu^{-2}+16 \pi^{2}}\right)$.

Test case I. Data on the inflow boundary satisfy the conditions for optimality (specifically, one measurement per grid node, which guarantees well-posedness, using the FE pair P1bubble-P1), IS internal sites are sparse in $\Omega$ and the reference distance is $l=1$, corresponding to half of the domain of interest.

Table 1 reports $E_{U}$ and $\alpha$, fixed empirically, for $H_{\mathrm{det}}, H_{\mathrm{ML}}$ and $H_{\mathrm{MAP}}$; with SNR set to 20 .

In this case, the ML estimator is well defined, since all the assumptions for the non singularity of the matrix in (2.14) hold; also, the ML estimate is the most accurate. If we look at the prior as a regularization term (in the deterministic sense), this result is somehow expected: system (2.14) is well-defined and regularization is not required. In the statistical perspective this means that when we have enough data, we do not need prior information. We notice however that the prior distribution used in our test cases is only based on regularity assumptions and not on real, known, statistical properties. In the follow up of the present work, we will repeat this analysis on real data sets. In this case, we can observe that the gain increases as we increase the number of internal data; the more noisy information we have, the better we can get from the statistical solution. Furthermore, the number of iterations required by the GMRESR for convergence is approximately 8 for both the approaches. 
TABLE 2. Accuracy results for statistical and deterministic solutions for the linearized NSE. On top, we consider a regularized minimization, comparing deterministic and MAP estimates. On bottom, we consider regularization by interpolation and compare the deterministic vs. the modified ML estimator (using regularization by inetrpolation and the pseudo-inverse of the noise).

\begin{tabular}{|l|c|c|c|c|}
\hline SNR & $\alpha$ & $\bar{E}_{U, \text { det }}$ & $\bar{E}_{U, \mathrm{MAP}}$ & $\gamma$ \\
\hline 20 & 0.5 & 0.0665 & 0.0530 & $24 \%$ \\
\hline 20 & 0.05 & 0.0666 & 0.0550 & $17 \%$ \\
\hline 20 & 0.005 & 0.0706 & 0.0579 & $18 \%$ \\
\hline 10 & 0.5 & 0.1272 & 0.0946 & $26 \%$ \\
\hline 10 & 0.05 & 0.1514 & 0.1032 & $32 \%$ \\
\hline 10 & 0.005 & 0.1256 & 0.1059 & $28 \%$ \\
\hline
\end{tabular}

\begin{tabular}{|c|c|c|c|}
\hline SNR & $\bar{E}_{U, \text { det }}$ & $\bar{E}_{U, \mathrm{ML}},(\mathrm{mod})$ & $\gamma$ \\
\hline 20 & 0.0709 & 0.0552 & $22 \%$ \\
\hline 10 & 0.1518 & 0.1256 & $17 \%$ \\
\hline
\end{tabular}

Test case II. In this case, using the same analytic solution and reference distance $l=1$, we consider data on the inflow boundary not satisfying sufficient conditions for optimality and additional internal data located on internal slices parallel to $\Gamma_{\mathrm{in}}$. This slice-wise location of the data - that resembles the position in Figure 2 and is a mandatory assumption in [16] - is however not strictly necessary for our methodology. We compare statistical and deterministic estimators and investigate the role of interpolation.

We first focus on the regularized case without interpolation and we compare $H_{\mathrm{MAP}}$ and $H_{\mathrm{det}}$. The ML estimator is in this case not well defined. The regularization parameter $\alpha$ is strongly data dependent; since we want to test the estimators over a set of noise realizations, finding the optimal $\alpha$ would be highly time consuming. For this reason, we compare deterministic and statistical approaches in correspondence of three fixed values of $\alpha$.

In Table 2 (top) we report relative errors $\bar{E}_{U}$, for $H_{\text {det }}$ and $H_{\mathrm{MAP}}$ in correspondence of $\alpha=0.5,0.05,0.005$. In this case we consider 300 internal data distributed on 10 slices (30 sites per slice, not in correspondence of grid nodes); also, SNR $=20$ and 10 , the number of noise realizations is $n=20$, the discretization step $\Delta=0.076$, and the number of sites on $\Gamma_{\text {in }}$ is $N_{s, \text { in }}=14$.

As expected, compared to the deterministic estimator, $H_{\mathrm{MAP}}$ is always more accurate since it takes into account additional information brought by statistical properties of the data. We stress that, since synthetic data are generated using the matrix $\mathrm{G}$, the noise correlation matrix in this case is exact.

In average the number of GMRESR iterations is higher for $H_{\mathrm{MAP}}(0.3$ times bigger than the one required by $\left.H_{\text {det }}\right)$; this is due to the presence of $\Sigma_{\text {noise }}^{-1}$ in the formulation.

In Table 2 (bottom) we report results obtained in correspondence of the same 20 noise realizations used in the regularized case, when the pseudo-inverse $\Sigma_{\eta}^{+}$is used. The modifed ML estimator is well defined and the additional knowledge brought by the noise correlation matrix makes it more accurate than $H_{\text {det }}$. On the other hand, the number of iterations in solving for $H_{\mathrm{ML} \text {,mod }}$ is 0.5 times bigger than the one required by $H_{\text {det }}$. As mentioned above, the higher number of iterations is expected and it is due to the presence of $\Sigma_{\eta}^{+}$. The additional effort is however balanced by the improved accuracy of the estimate.

Test case III. We present numerical results obtained solving the nonlinear NSE using the same analytic solution and reference distance of Test case I and II. We consider data on $\Gamma_{\text {in }}$ not satisfying sufficient conditions for optimality and internal data located on 10 internal slices (with $30 \mathrm{data} / \mathrm{slice}$ ). We solve iteratively (1.5) with $w=0.5$ and $\vartheta=0.2$. As in the previous cases, the number of iterations is related to the presence of the noise correlation matrix; the statistical approach is 0.4 times more expensive than the deterministic one. In Table 3 
TABLE 3. Accuracy results for statistical and deterministic solutions for the nonlinear NSE.

\begin{tabular}{|l|c|c|c|c|c|c|c|}
\hline \multicolumn{4}{|c|}{ MAP, $\alpha=0.5$} & \multicolumn{4}{|c|}{ ML } \\
\hline SNR & $\bar{E}_{U, \text { det }}$ & $\bar{E}_{U, \text { MAP }}$ & $\gamma$ & SNR & $\bar{E}_{U, \text { det }}$ & $\bar{E}_{U, \mathrm{ML}}$ & $\gamma$ \\
\hline 20 & 0.0822 & 0.07371 & $10 \%$ & 20 & 0.0855 & 0.0579 & $6 \%$ \\
\hline 10 & 0.1394 & 0.1041 & $25 \%$ & 10 & 0.1675 & 0.1363 & $18 \%$ \\
\hline
\end{tabular}

TABLE 4. Accuracy results for statistical and deterministic solutions for the axisymmetric case.

\begin{tabular}{|l|c|c|c|}
\hline SNR & $\bar{E}_{U, \text { det }}$ & $\bar{E}_{U, \mathrm{MAP}}$ & $\gamma$ \\
\hline 20 & 0.0396 & 0.0308 & $22 \%$ \\
\hline 10 & 0.1423 & 0.0978 & $31 \%$ \\
\hline
\end{tabular}

we report results obtained in correspondence of $\Delta=0.076$ and SNR $=20$ and 10 . In the computation of $H_{\mathrm{MAP}}$ the regularization parameter is chosen according to the results obtained in the linearized case; we choose $\alpha=$ 0.5 since it corresponds to the most accurate numerical solution. The poor gain in correspondence of SNR $=$ 20 means that statistical information associated with a low amount of noise is not significant enough to make a considerable difference with respect to deterministic estimates in terms of accuracy. In the other cases statistical estimators are significantly more accurate and yield a gain up to the $25 \%$ in correspondence of low values of SNR.

Test case IV. We consider an axisymmetric formulation for the numerical solution of the non linear NSE, corresponding to the 3 -dimensional Poiseuille flow in a cylinder of length $L=6$, diameter $D=3$ and viscosity $\nu=1$ in a 2 -dimensional structured grid of dimension $80 \times 20$. Data on the inflow boundary do not satisfy sufficient conditions and internal data are located on 5 internal slices with 10 data per slice. Here, the reference distance is $l=1.5$ (corresponding to one half of the diameter). We perform interpolation and, in order to prevent potential ill-conditioning, we add a regularization term (or smoothness prior) with $\alpha=1 \mathrm{e}-7$. Results for SNR $=$ 20 and 10 are reported in Table 4; in both cases we have a significant gain in accuracy. Also, the number of iterations, in average, is the same.

\subsection{Spread estimators}

Test case I. We consider the square geometry $[-0.5,1.5] \times[0,2]$ and the analytic solution (3.2); data on $\Gamma_{\text {in }}$ satisfy conditions for optimality in Proposition 1.1. For this test case the number of degrees of freedom of the forward problem is sufficiently small to allow explicit computation and storage of correlation matrices; matrix operations are performed in Matlab. In particular, for the computation of $\Sigma_{\text {post }}^{-1}=\Sigma_{\text {pr }}^{-1}+\mathrm{Z}^{\mathrm{T}} \Sigma_{\text {noise }}^{-1} \mathrm{Z}$ and $\Sigma_{U}=\mathrm{T} \Sigma_{\text {post }}^{-1} \mathrm{~T}^{\mathrm{T}}$ we use the conjugate gradient method. For each sub matrix of $\Sigma_{U}$ defined as in (2.16), corresponding to horizontal and vertical velocities on one DOF, say the $j$ th DOF, we compute the square root of the spectral radius, $\lambda_{\max , \mathrm{j}}$; as described in Section 2.3 this is the maximum deviation from the mean in a $60 \%$ confidence region. In Figure 4 (top left) we report the map of $\sqrt{\lambda_{\max , \mathrm{j}}}$ over the computational grid for $\mathrm{SNR}=20$. We observe a peak on the inflow boundary ${ }^{5}$, this is due to the fact that the noise of data on $\Gamma_{\text {in }}$ has high influence on the solution, the result is a higher uncertainty for the velocity components. In correspondence of three areas featuring different values of deviation we compute a $80 \%$ confidence region (see Fig. 4 top right and bottom) corresponding to the set

$$
\left(\mathbf{U}_{j}-U_{j}\right)^{\mathrm{T}} \Sigma_{U}^{-1}\left(\mathbf{U}_{j}-U_{j}\right) \leq \chi_{2}^{2}(0.2) \cong 3.219,
$$

where $\mathbf{U}_{j}$ corresponds to the vector of horizontal and vertical velocities on the $j$ th DOF and $U_{j}$ is the corresponding mean value. $\mathbf{U}_{e x}$ in the figures is the exact velocity on the $j$ th DOF, note that it always falls

\footnotetext{
${ }^{5}$ Results are more readable in the colored picture of the electronic version of the paper.
} 
TABLE 5. Accuracy results and maximum deviation for different values of SNR.

\begin{tabular}{|l|c|c|c|}
\hline \multicolumn{4}{|c|}{ Test case I } \\
\hline SNR & $\alpha$ & $E_{U, \mathrm{MAP}}$ & $\lambda_{\mathrm{MAX}}$ \\
\hline 20 & 0.5 & 0.06003 & 0.09759 \\
10 & 0.5 & 0.07754 & 0.1744 \\
\hline
\end{tabular}

\begin{tabular}{|c|c|c|c|}
\hline \multicolumn{4}{|c|}{ Test case II } \\
\hline SNR & $\alpha$ & $E_{U, \text { MAP }}$ & $\lambda_{\text {MAX }}$ \\
\hline 20 & $1 . e-5$ & 0.01720 & 0.3759 \\
10 & $1 . e-5$ & 0.02565 & 0.7024 \\
\hline
\end{tabular}
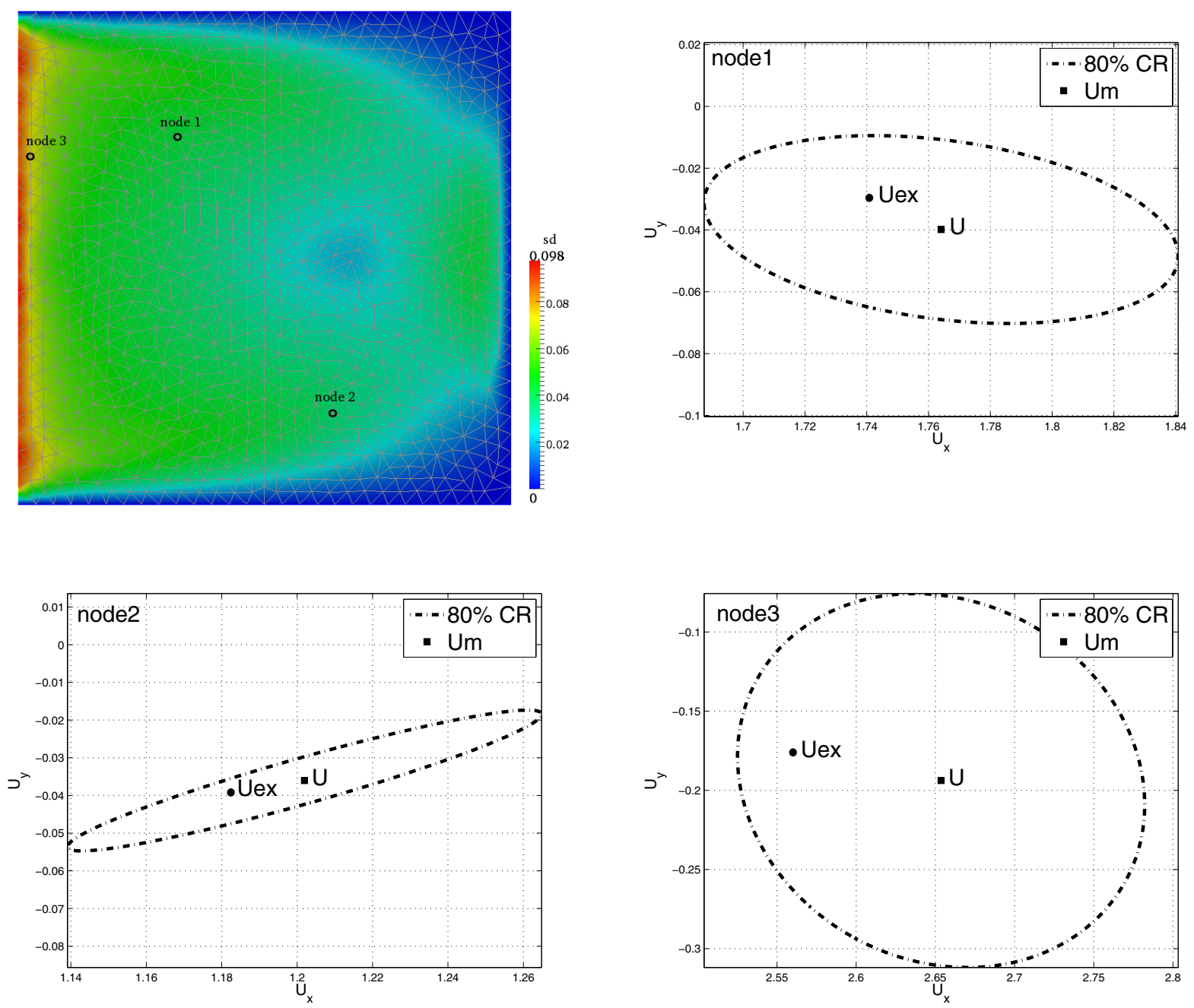

Figure 4. Map of the maximum deviation from the mean of the velocity for Test case I (top left) and ellipses corresponding to node 1 (top right), 2 (bottom left) and 3 (bottom right).

inside the ellipsis of confidence. Results for SNR $=20$ and SNR $=10$ are reported in Table 5 (left). Here $\lambda_{\text {MAX }}=\max _{j}\left(\lambda_{\max }, j\right)$ is the maximum deviation over the computational grid.

Even though not realistic, this test case highlights the fact that Bayesian inversion reduces the standard deviation of the noise affecting observed measures, 0.1467 for SNR $=20$ and 0.2953 for $\mathrm{SNR}=10$. 


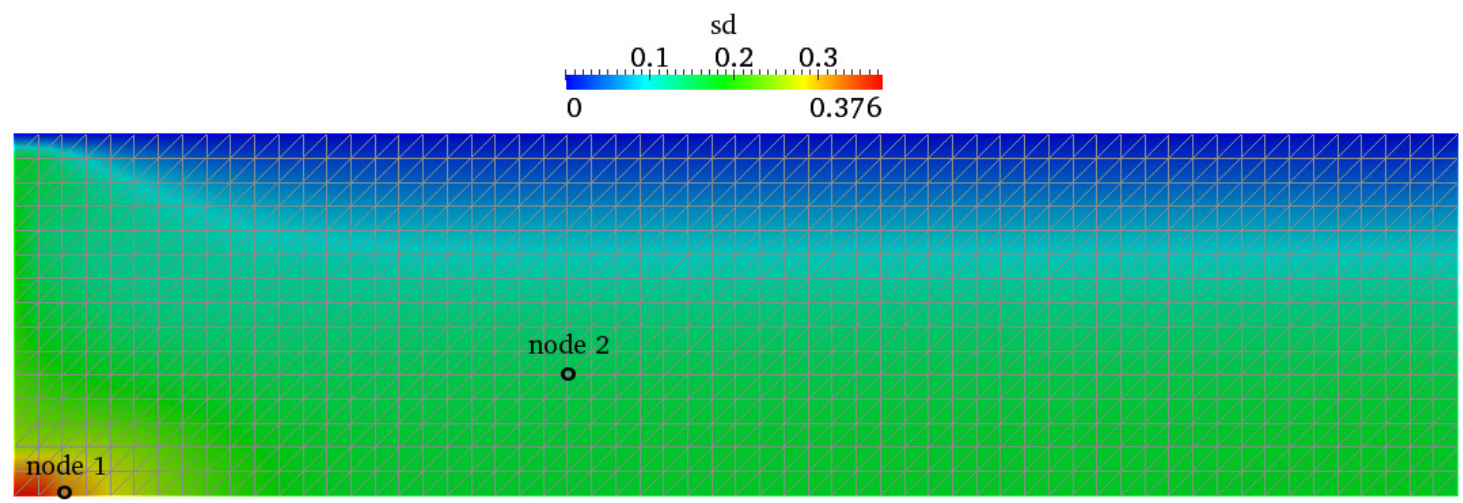

FiguRE 5. Map of the maximum deviation from the mean of the velocity for Test case II.
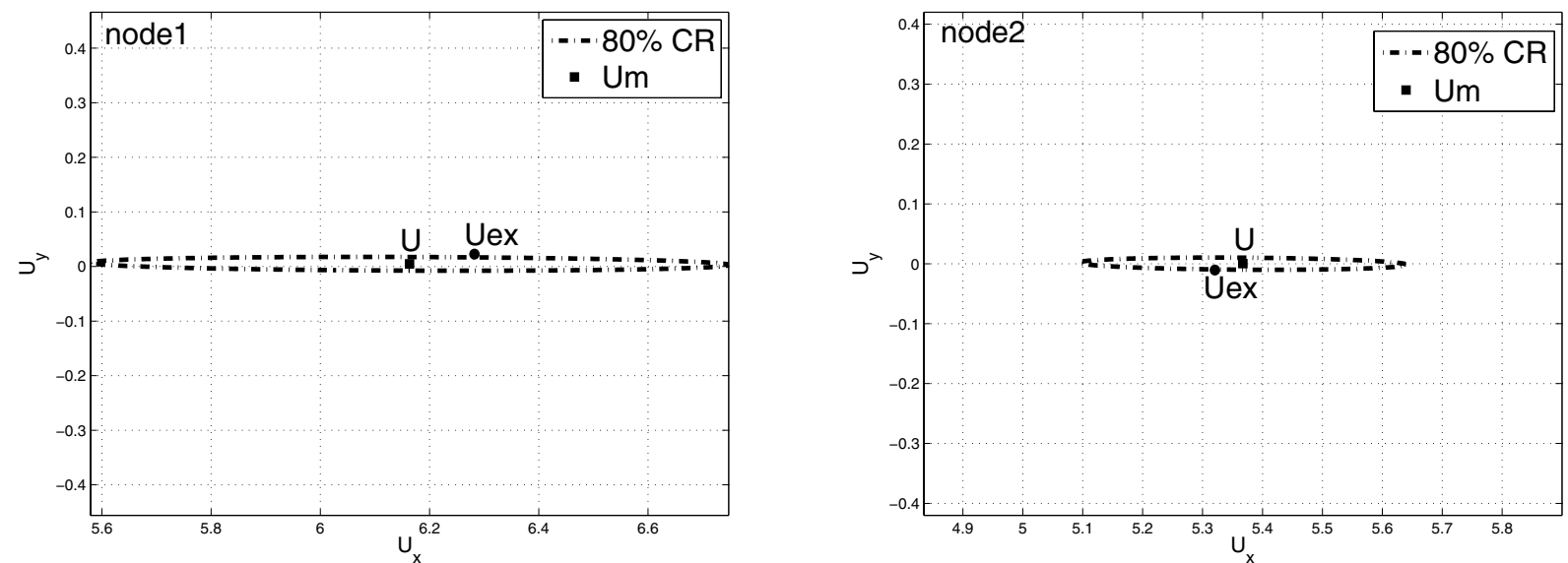

FiguRE 6. Ellipses corresponding to node 1 (left) and 2 (right).

Test case II. We consider the 3 -dimensional axisymmetric formulation and data on $\Gamma_{\text {in }}$ not satisfying conditions for optimality; thus, we perform interpolation of the available data. Recall that, in this case, $\Sigma_{\text {noise }}=\Pi \Sigma_{\varepsilon} \Pi^{\mathrm{T}}$, where $\Sigma_{\varepsilon}$ is the correlation matrix for the measures. Matrices operations are treated as in the previous test case. In Figure 5 we report the map of $\sqrt{\lambda_{\max , j}}$ in each DOF for SNR $=20$. Here, the peak is located in the lower left corner ${ }^{6}$. This result is related to boundary conditions. In fact, on the wall and on the outflow section we prescribe exact boundary data; for this reason, we do not have uncertainty. As we move far from such boundaries, i.e. towards the axis of symmetry and the inflow section we observe a higher deviation from the mean. This behavior is emphasized by the fact that the boundary condition on $\Gamma_{\text {in }}$ is the control variable which is highly affected by the measurement noise. Nevertheless, we observe that except for the bottom left corner the deviation is lower that the one of the data, namely 0.325 for SNR $=20$ and 0.65 for $\mathrm{SNR}=10$. As in Test case I, we compute the $80 \%$ confidence region in areas featuring different behavior. Results for SNR $=20$ are reported in Figure 6; note that in this case we have a horizontal flow, this explains the low deviation from the mean for vertical components of the velocity.

\footnotetext{
${ }^{6}$ Results are more readable in the colored picture of the electronic version of the paper.
} 

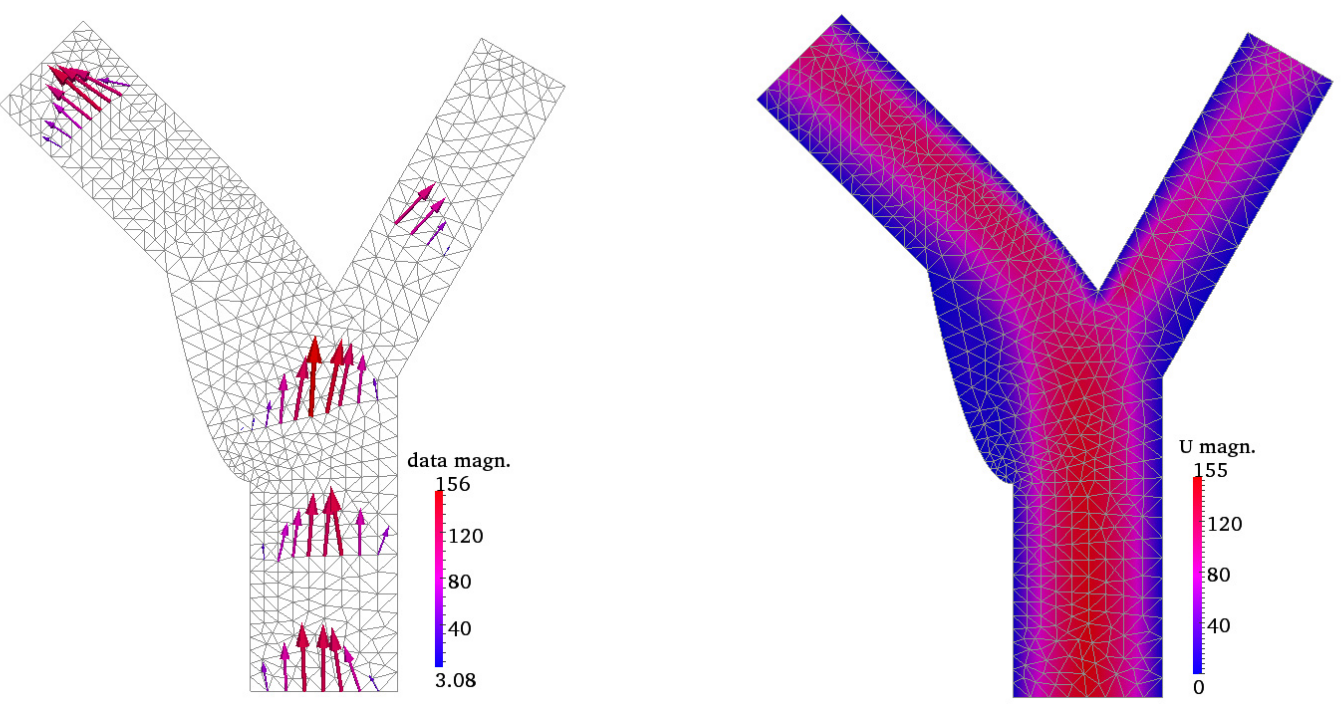

FiguRE 7. Data configuration and magnitude of the velocity field.

TABLE 6. Comparison results between deterministic and ML estimator: accuracy with respect to the reference solution.

\begin{tabular}{|l|c|c|c|c|}
\hline SNR & $n$ & $\bar{E}_{U, \text { det }}$ & $\bar{E}_{U, \mathrm{ML}}$ & $\gamma$ \\
\hline 50 & 20 & 0.02951 & 0.02212 & $25 \%$ \\
\hline 20 & 20 & 0.05273 & 0.03617 & $31 \%$ \\
\hline 10 & 20 & 0.11230 & 0.06600 & $41 \%$ \\
\hline
\end{tabular}

\subsection{Towards real geometries}

In this section we consider a more complex geometry. We reproduce a 2-dimensional section of the carotid. This test case has already been considered in [7] in the deterministic framework; here, we compare statistical and deterministic estimators and we compute confidence intervals for the velocity and for the WSS.

Location of the data is reported in Figure 7 (left), internal data lie on four layers in correspondence of grid nodes. These are generated adding Gaussian noise to a reference solution $\mathbf{U}_{\mathrm{FE}}$, which is the FE solution of the forward problem on a very fine grid (300\% of the grid nodes used in this test case). The correlation matrix for the Gaussian noise is defined as in equation (3.1). The indexes of accuracy are the average relative error $\bar{E}_{U}=\frac{1}{n} \sum_{i=1}^{n} E_{U, i}$ where $E_{U, i}$ is associated with the $i$ th realization and it is defined as $E_{U}=\frac{\left\|\mathbf{U}-\mathbf{U}_{\mathrm{FE}}\right\|_{2}}{\left\|\mathbf{U}_{\mathrm{FE}}\right\|_{2}}$, and the gain $\gamma=1-\frac{\bar{E}_{U, \text { stat }} \text {. }}{\bar{E}_{U, \text { det }}}$.

In Table 6 we report comparison results between statistical and deterministic estimators; since data satisfy conditions for optimality we set the regularization parameter $\alpha=0$, which corresponds to computing the ML estimator in the statistical formulation. These results show that also for more complex geometries statistical estimators are robust with respect to the noise and they are significantly more accurate than the deterministic ones. As en example, in Figure 7 we report the magnitude of the assimilated velocity field; in Figure 8 (left) the difference between deterministic and reference solution, and between statistical and reference solution in the area close to the inflow boundary. Here, the statistical solution is more accurate. The ML estimator is less sensitive to the noise that significantly affects the numerical solution on $\Gamma_{\mathrm{in}}$. 

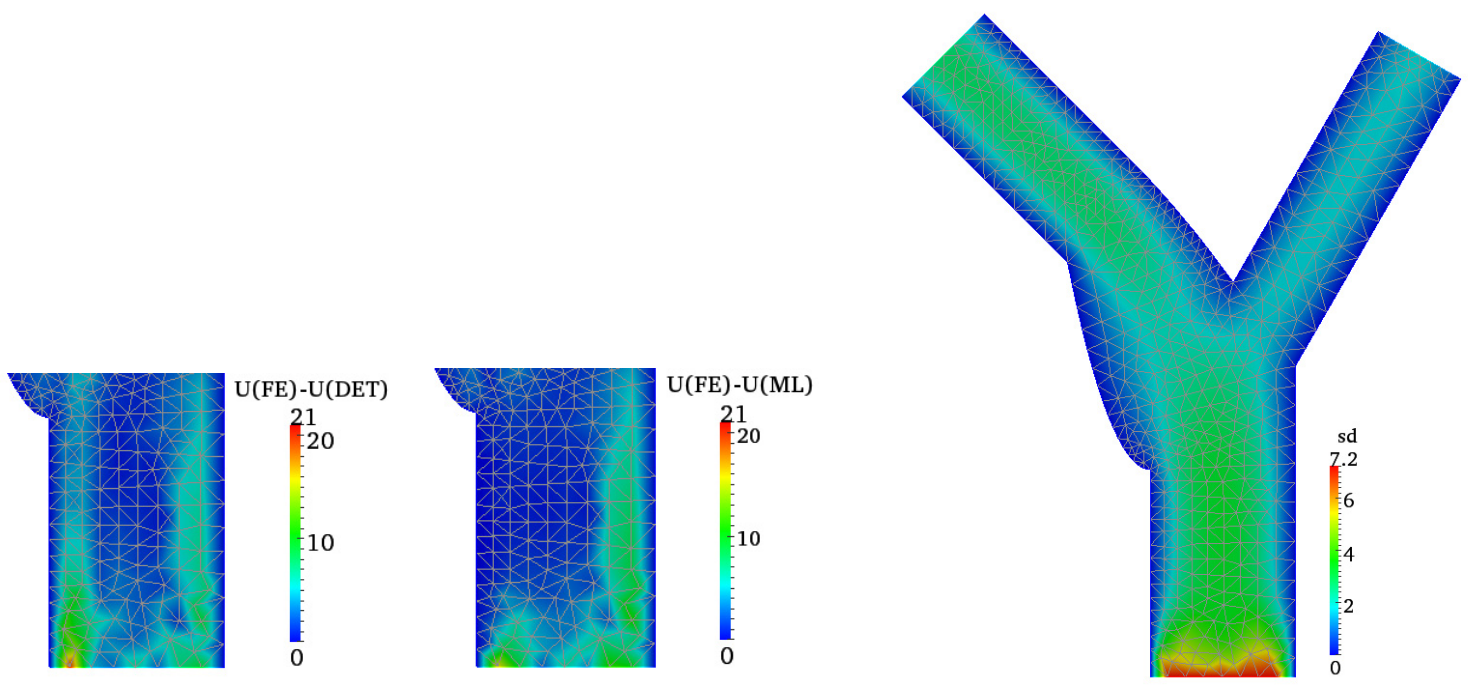

FIGURE 8. On the left: comparison results between deterministic and ML estimator with SNR = 20: map of the absolute error between deterministic and reference solution and between statistical and reference solution. On the right: map of the maximum deviation from the mean of the velocity.

In view of the application to real cardiovascular problems we want to estimate the confidence intervals for the velocity. In Figure 8 (right) we report the map of $\sqrt{\lambda_{\max }}$ for SNR $=20$ over the computational grid; consistently with previous results we observe that the standard deviation increases far from the wall since we work in a rigid assumption and we prescribe homogeneous (exact and noise-free) Dirichlet conditions on the walls. Furthermore, since close to $\Gamma_{\text {in }}$ the solution is more sensitive to the noise in this area we have the highest deviation from the mean.

The wall shear stress As we have pointed out previously, in hemodynamics it is fundamental to estimate other flow related variables as the WSS since, as a matter of fact, they are crucial for predicting the occurrence of diseases or even the rupture of a blood vessel.

In medical practice [8] the WSS is computed using the observed velocity data (either projected onto the whole grid or collected on each node); these estimators are not accurate enough and are more sensitive to the presence of the noise. Using point and statistical estimators for the velocity we compute statistical parameters of the WSS distributions; also in this case we assume this random vector to be normally distributed: WSS $\mathcal{N}\left(W S S, \Sigma_{W S S}\right)$, mean and correlation matrix are determined by the PDF of the velocity. In fact, velocity and WSS are related by the linear transformation

$$
\mathbf{W S S}=\mathrm{T}_{w} \mathbf{U}
$$

where $\mathrm{T}_{w} \in \mathbb{R}^{N_{u}, N_{w}}$ maps the discretized velocity into the discretized WSS; $N_{w}$ being the number of DOFs of the WSS. According to property $\mathbf{P} 1$ in Appendix WSS has a Gaussian distribution with mean $W S S=\mathrm{T}_{w} U$ and covariance $\Sigma_{w}=\mathrm{T}_{w} \Sigma_{U} \mathrm{~T}_{w}^{\mathrm{T}}$. Furthermore, according to $\mathbf{P 2}$, the marginal distribution of the vector of horizontal and vertical components of the WSS on each DOF is defined as

$$
\mathcal{N}\left(\left[\begin{array}{l}
W S S_{i} \\
W S S_{i+N_{w} / 2}
\end{array}\right],\left[\begin{array}{ll}
\Sigma_{W S S ; i, i} & \Sigma_{W S S ; i, i+N_{u} / 2} \\
\Sigma_{W S S ; i+N_{u} / 2, i} & \Sigma_{W S S ; i+N_{u} / 2, i+N_{u} / 2}
\end{array}\right]\right) .
$$

Figure 9 (left) refers to the WSS on the left inner wall of the bifurcation; here, the magnitude of the vector in each DOF is displayed as a function of the horizontal coordinate. In this case, the relative error, in norm $l_{2}$, with 

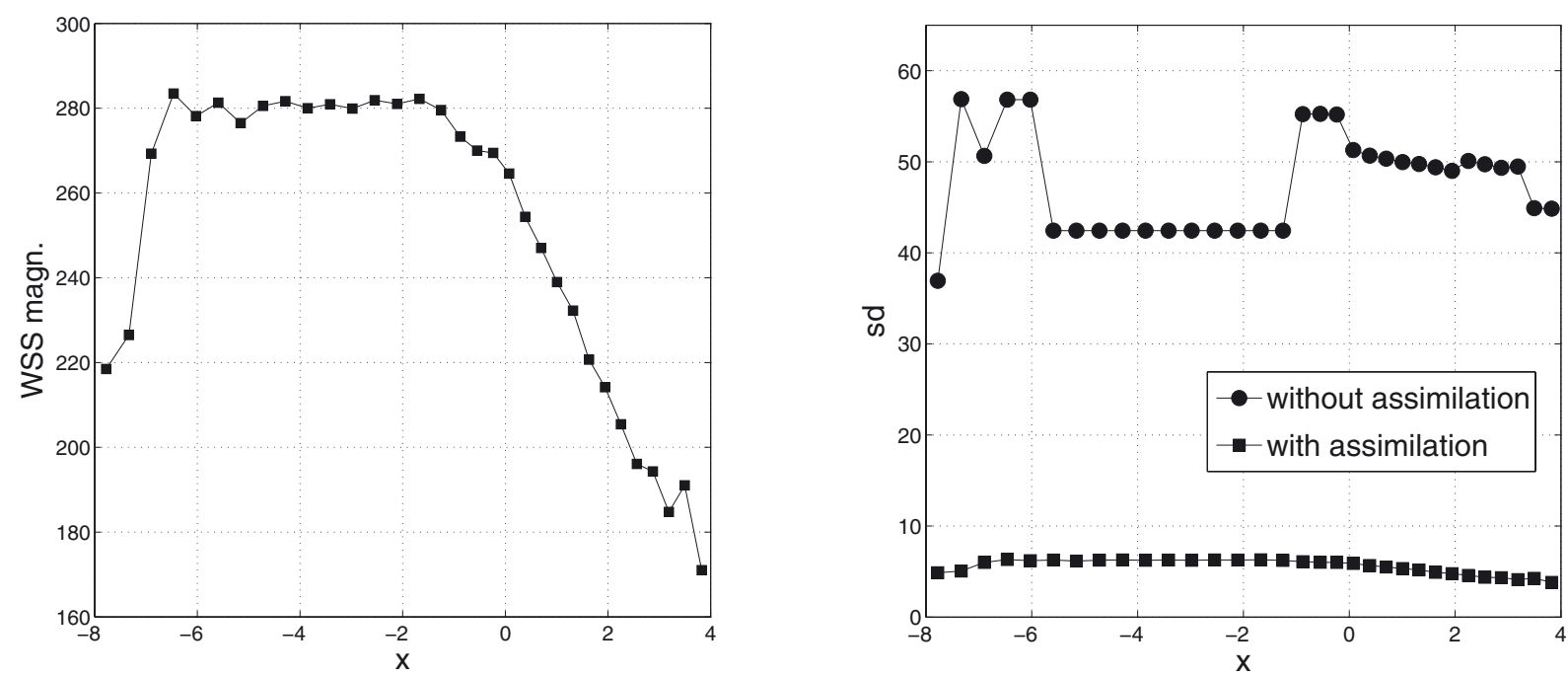

Figure 9. On the left, WSS on a selected wall: magnitude as a function of the $x$-coordinate. On the right, standard deviation of the assimilated and non-assimilated WSS as a function of the $x$-coordinate.

respect to the reference solution is of 0.1483 with $\mathrm{SNR}=20$. We notice that for unsteady problems the most interesting part of the carotid wall is on the external side of the left branch (inner carotid artery). However, in the steady case we found more significant results on the internal side.

As we did for the velocity, we compute in each DOF $\sqrt{\lambda_{\max }}$. In Figure 9 (right) we report, on such selected wall, the value of the "assimilated" $\sqrt{\lambda_{\max }}$ for each DOF together with the same quantity for a "non-assimilated" WSS. More specifically, in the second case, we assume WSS $=\mathrm{T}_{w} \mathbf{U}_{\text {obs }}$, where $\mathbf{U}_{\text {obs }}$ is the vector of observed velocities. Thus, it features the correlation matrix

$$
\left[\Sigma_{\mathrm{obs}}\right]_{i j}=\exp \left\{-\frac{1}{l^{2}}\left\|\mathbf{x}_{i}^{m}-\mathbf{x}_{j}^{m}\right\|_{2}^{2}\right\}
$$

where $\mathbf{x}_{i}^{m}, i=1, \ldots N_{u}$, are observation sites (here corresponding to DOFs of the velocity). According to these relations, WSS $\sim \mathcal{N}\left(\mathrm{T}_{w} U_{\mathrm{obs}}, \Sigma_{\mathrm{obs}}\right)$. From the results we note that the maximum deviation from the mean is much higher than the assimilated one; note that the flat configuration of the non-assimilated WSS in the interval $(-6,-1)$ is due to the grid-structure, very regular in correspondence of that segment. In fact, the standard deviation is due to the $T_{w}$ map and the data correlation matrix. The first depends on the elements area and the second on the mutual distance between grid nodes. The structured configuration of the mesh in this area makes the standard deviation constant. Even if the result is preliminary and depends on the data configuration, the huge reduction in the standard deviation obtained using DA, lead us to conclude that we can effectively filter the noise.

\section{Conclusion}

Motivated by hemodynamics applications we introduced an assimilation technique for the estimation of statistical properties of the velocity field for fluid dynamics simulations. The method is based on the Bayesian approach to inverse problems and can be formulated as an optimization problem where a weighted misfit between velocity and data is minimized under the constraint of the NSE. An important aspect that makes our technique valuable is the inclusion of the additional knowledge regarding the measurement process and the statistical 
features of the measurement noise. When data are characterized by high correlation (which is very likely in case of complex systems as the cardiovascular one) this significantly determines the statistical properties of the flow and affects the quality of the assimilation process.

Numerical results show the noise filtering properties of the method. If we focus on the case of the carotid (Sect. 3.4), we observe that the inclusion of statistical information, such as the data correlation function, in the solution of the inverse problem results in a considerable gain in accuracy (up to the 30\%) with respect to the formulation introduced in [7]. Furthermore, the analysis of the standard deviation of the velocity reveals that the deviation from the mean of the assimilated solution is at most half the one of the given data, apart from the region at the inflow, where the noise has a a major impact. This fact implies that the uncertainty of the WSS computed using the assimilated solution is significantly lower than the one obtained using given data only.

Although promising and potentially of great impact, this assimilation technique is still at its beginning stage and has some limitations. For the DA estimator to be applicable to 3-dimensional unsteady test cases with real geometries and data several computational issues have to be considered in order to improve the efficiency of the method. Future work includes the employment of different preconditioners for the state and adjoint systems, which are the bottleneck of the numerical solution. For the solution of the nonlinear optimization problem we propose a nonlinear procedure that stems from the combination of the DA method for linear problems and fixed point iterative methods for the NSE. In literature there are plenty of methods for the solution of problems of this sort. For reasons related to the FE library, lifeV, that we utilize we plan to adopt the NOX package ${ }^{7}$, which is part of Sandia's Trilinos project. Another important issue is to be competitive with other assimilation approaches, such as the Kalman filter methods [1]. A way to speed up the computation and to bring these methodologies to end-user applications is to combine efficient optimization strategies with model reduction (this is the subject of our current research). In doing this it is important to find a good compromise between accuracy and efficiency; in medicine this is a delicate issue. Several methods are available, among these we mention the reduced basis method for parameter-based reduction and the proper orthogonal decomposition [13, 26]. In a long term perspective, the approaches of [25], for the Young modulus identification, and of the present paper should be merged to obtain a simultaneous filtering and identification.

Acknowledgements. The authors would like to thank Dr. M. Perego for his suggestions, inspiring ideas and fruitful discussions. The authors also thank the referees for their careful reading of this paper and for their many suggestions that resulted in substantial improvements.

\section{Appendix A. Multivariate normal And Chi-SQuared Distributions}

In this section we report basic concepts regarding the normal distribution for random vectors and we introduce properties and results specifically related to our application, for more details see e.g. [3,28].

\section{The multivariate normal PDF}

The multivariate normal distribution is a generalization to dimensions $d \geq 2$ of the univariate normal distribution. Let $\mathbf{X}$ be a $d$-dimensional random vector. The Gaussian multivariate distribution reads

$$
f(\mathbf{x})=\frac{1}{\sqrt{(2 \pi)^{d} \operatorname{det}(\Sigma)}} \exp \left\{-(\mathbf{x}-\boldsymbol{\mu})^{\mathrm{T}} \Sigma^{-1}(\mathbf{x}-\boldsymbol{\mu})\right\} \quad \forall \mathbf{x} \in \mathbb{R}^{d},
$$

denoted by $\mathbf{X} \sim \mathcal{N}(\boldsymbol{\mu}, \Sigma)$. Here $\boldsymbol{\mu} \in \mathbb{R}^{d}$ represents the expected value of the multivariate random variable $\mathbf{X} ;$ it is the point of maximum density, mode and expected value [18]. $\Sigma$ is the symmetric positive definite covariance matrix of $\mathbf{X}$, i.e. $\Sigma_{i, i}=\operatorname{var}\left(\mathbf{X}_{i}\right)$ and $\Sigma_{i, j}=\Sigma_{j, i}=\operatorname{cov}\left(\mathbf{X}_{i}, \mathbf{X}_{j}\right)$ for $i, j=1, \ldots, d$.

\footnotetext{
${ }^{7}$ http://trilinos.sandia.gov/packages/nox/
} 
Contours of constant density play a central role in determining confidence regions for multivariate normal variables. For the $d$-dimensional distribution these are ellipsoids generated by the equation

$$
(\mathbf{x}-\boldsymbol{\mu})^{\mathrm{T}} \Sigma^{-1}(\mathbf{x}-\boldsymbol{\mu})=c^{2} ;
$$

these ellipsoids are centered in $\boldsymbol{\mu}$ and have axes $\pm c \sqrt{\lambda_{j}} \mathbf{e}_{j}$, where $\lambda_{j}$ are the eigenvalues of $\Sigma$ and $\mathbf{e}_{j}$ the associated eigenvectors for $j=1, \ldots, d$.

\section{The chi-squared distribution}

A scalar random variable $Z$ has a chi-square distribution with $d$ degrees of freedom $\left(Z \sim \chi_{d}^{2}\right)$ when its $\mathrm{PDF}$ is given by

$$
f(z ; d)= \begin{cases}\frac{1}{2^{d / 2} \Gamma(d / 2)} z^{d / 2-1} \mathrm{e}^{-z / 2}, & z \geq 0 ; \\ 0, & \text { otherwise }\end{cases}
$$

where $\Gamma$ denotes the Gamma function defined as $\Gamma\left(\frac{n}{2}\right)=\sqrt{\pi} \frac{(n-2) ! !}{2^{(n-1) / 2}}$ for a half-integer $\frac{n}{2}$.

Properties of the multivariate normal distribution

For a $d$-dimensional normal random vector $\mathbf{X} \sim \mathcal{N}(\boldsymbol{\mu}, \Sigma)$ we have the following properties:

P1 Affine transformations of $\mathbf{X}$ are normally distributed. In particular, given $\mathrm{A} \in \mathbb{R}^{q \times d}$ and $\mathbf{b} \in \mathbb{R}^{q}$, then $\mathbf{Y}=\mathrm{AX}+\mathbf{b} \sim \mathcal{N}_{q}\left(\mathrm{~A} \boldsymbol{\mu}+\mathbf{b}, \mathrm{A} \Sigma \mathrm{A}^{\mathrm{T}}\right)$.

P2 For all subsets of the components of $\mathbf{X}$ we can define a marginal distribution, still Gaussian. If we rearrange the vector as

$$
\mathbf{X}=\left[\begin{array}{l}
\mathbf{X}_{1} \\
\mathbf{X}_{2}
\end{array}\right], \quad \boldsymbol{\mu}=\left[\begin{array}{l}
\boldsymbol{\mu}_{1} \\
\boldsymbol{\mu}_{2}
\end{array}\right], \quad \Sigma=\left[\begin{array}{ll}
\Sigma_{1,1} & \Sigma_{1,2} \\
\Sigma_{2,1} & \Sigma_{2,2}
\end{array}\right]
$$

where $\mathbf{X}_{1} \in \mathbb{R}^{q}$ and $\mathbf{X}_{2} \in \mathbb{R}^{d-q}$; then, the marginal distribution of $\mathbf{X}_{1}$ is $\mathcal{N}_{q}\left(\boldsymbol{\mu}_{1}, \Sigma_{1,1}\right)$ and the one of $\mathbf{X}_{2}$ is $\mathcal{N}_{d-q}\left(\boldsymbol{\mu}_{2}, \Sigma_{2,2}\right)$.

P3 $(\mathbf{X}-\boldsymbol{\mu})^{\mathrm{T}} \Sigma^{-1}(\mathbf{X}-\boldsymbol{\mu})$ is distributed as $\chi_{d}^{2}[18]$.

By definition, this means that if $\xi$ denotes the upper $(100 \xi)$ th percentile of the $\chi_{d}^{2}$ distribution, the probability of the vector $\mathbf{x}$ to belong to the ellipsoid $\left\{\mathbf{x}:(\mathbf{x}-\boldsymbol{\mu})^{\mathrm{T}} \Sigma^{-1}(\mathbf{x}-\boldsymbol{\mu}) \leq \chi_{d}^{2}(\xi)\right\}$ is $1-\xi$.

\section{REFERENCES}

[1] C. Bertoglio, P. Moireau and Jean-Frédéric Gerbeau, Sequential parameter estimation for fluid-structure problems. Application to hemodynamics. Inter. J. Numer. Methods Biomed. Eng. 28 (2012) 434-455. RR-7657.

[2] J. Blum, F.X. Le Dimet and I.M. Navon, Data Assimilation for Geophysical Fluids, Handbook of numerical analysis, vol. XIV, chapter 9. Elsevier (2005).

[3] D.C. Boes, FA Graybill and A.M. Mood, Introduction to the Theory of Statistics. McGraw-Hill (1974).

[4] D. Calvetti and E. Somersalo, Subjective knowledge or objective belief? an oblique look to bayesian methods, in Large-Scale Inverse Problems and Quantification of Uncertainty, edited by G. Biros et al. Wiley Online Library (2011) 33-70.

[5] M. D'Elia, Ph.D. thesis.

[6] M. D'Elia, L. Mirabella, T. Passerini, M. Perego, M. Piccinelli, C. Vergara and A. Veneziani, Applications of Variational Data Assimilation in Computational Hemodynamics, chapter 12. MS \& A. Springer (2011) 363-394.

[7] M. D'Elia, M. Perego and A. Veneziani, A variational Data Assimilation procedure for the incompressible Navier-Stokes equations in hemodynamics. Technical Report TR-2010-19, Department of Mathematics and Computer Science, Emory University, To appear in J. Sci. Comput. Available on www.mathcs.emory.edu (2010).

[8] P.M. den Reijer, D. Sallee, P. van der Velden, E. Zaaijer, W.J. Parks, S. Ramamurthy, T. Robbie, G. Donati C. Lamphier, R. Beekman and M. Brummer, Hemodynamic predictors of aortic dilatation in bicuspid aortic valve by velocity-encoded cardiovascular magnetic resonance. J. Cardiovasc. Magn. Reson. 12 (2010) 4.

[9] H.A. Van der Vorst and C. Vuik, Gmresr: a family of nested gmres methods. Numer. Linear Algebra Appl. 1 (1994) 369-386.

[10] R.P. Dwight, Bayesian inference for data assimilation using Least-Squares Finite Element methods, in IOP Conf. Ser. Mat. Sci. Eng., vol. 10. IOP Publishing (2010) 012224.

[11] L. Formaggia, A. Veneziani and C. Vergara. SIAM J. Sci. Comput. (2008).

[12] L. Formaggia, A. Veneziani and C. Vergara. Comput. Methods Appl. Mech. Eng. (2010). 
[13] M. Frangos, Y. Marzouk, K. Willcox and B. van Bloemen Waanders, Surrogate and reduced-order modeling: A comparison of approaches for large-scale statistical inverse problems. Large-Scale Inverse Problems and Quantification of Uncertainty (2010) 123-149.

[14] M.D. Gunzburger, Perspectives in flow control and optimization. Society for Industrial Mathematics 5 (2003).

[15] Per Christian Hansen, Rank-deficient and discrete ill-posed problems. SIAM Monographs on Mathematical Modeling and Computation. Society for Industrial and Applied Mathematics (SIAM), Philadelphia, PA (1998).

[16] J.J. Heys, T.A. Manteuffel, S.F. McCormick, M. Milano, J. Westerdale and M. Belohlavek, Weighted least-squares finite elements based on particle imaging velocimetry data. J. Comput. Phys. 229 (2010) 107-118.

[17] J. G. Heywood, R. Rannacher and S. Turek, Artificial boundaries and flux pressure conditions for the incompressible navierstokes equations. Int. J. Numer. Methods Fluids 22 (1996) 325-352.

[18] R.A. Johnson and D.W. Wichern, Applied multivariate statistical analysis. Prentice-Hall, Inc., Upper Saddle River, NJ, USA (1988).

[19] J. Kaipio and E. Somersalo, Statistical and Computational Inverse Problems. Springer (2005).

[20] E.M. Kalman, A new approach to linear filtering and prediction problems. Trans. ASME-J. Basic Eng. 82 (1960) 35-45.

[21] D. Kay, D. Loghin and A. Wathen, A preconditioner for the steady-state navier-stokes equations. SIAM J. Sci. Comput. 24 (2002) 237-256.

[22] P. Moireau, C. Bertoglio, N. Xiao, C. Figueroa, C. Taylor, D. Chapelle and J.-F. Gerbeau, Sequential identification of boundary support parameters in a fluid-structure vascular model using patient image data. Biomechanics and Modeling in Mechanobiology. Published Online (2012) 1-22.

[23] P. Moireau and D. Chapelle, Reduced-order unscented kalman filtering with application to parameter identification in largedimensional systems. ESAIM: COCV 17 (2011) 380-405.

[24] J. Nocedal and S. Wright, Numerical Optimization. Springer (2000).

[25] M. Perego, A. Veneziani and C. Vergara, A variational approach for estimating the compliance of the cardiovascular tissue: An inverse fluid-structure interaction problem. SIAM J. Sci. Comput. 33 (2011) 1181-1211.

[26] A. Quarteroni, G. Rozza and A. Manzoni, Certified reduced basis approximation for parametrized partial differential equations and applications. J. Math. Ind. 1 (2011) 3.

[27] D. Silvester, H. Elman, D. Kay and A. Wathen, Efficient preconditioning of the linearized navier-stokes equations for incompressible flow. J. Comput. Appl. Math. 128 (2001) 261-279.

[28] A. Tarantola, Inverse problem theory and methods for model parameter estimation. Society for Industrial Mathematics (2005).

[29] A. Veneziani, Boundary conditions for blood flow problems, in Proc. of ENUMATH97, edited by R. Rannacher et al., World Sci. Publishing (1998).

[30] A. Veneziani, Mathematical and Numerical Modeling of Blood flow Problems. Ph.D. thesis, Politecnico di Milano, Italy (1998).

[31] C. Vuik, New insights in gmres-like methods with variable preconditioners. J. Comput. Appl. Math. 61 (1995) 189-204. 